\title{
EEG seizure detection and prediction algorithms: a survey
}

Turkey N Alotaiby ${ }^{1}$, Saleh A Alshebeili², Tariq Alshawi ${ }^{3}$, Ishtiaq Ahmad ${ }^{4}$ and Fathi E Abd El-Samie ${ }^{5,6^{*}}$

\begin{abstract}
Epilepsy patients experience challenges in daily life due to precautions they have to take in order to cope with this condition. When a seizure occurs, it might cause injuries or endanger the life of the patients or others, especially when they are using heavy machinery, e.g., deriving cars. Studies of epilepsy often rely on electroencephalogram (EEG) signals in order to analyze the behavior of the brain during seizures. Locating the seizure period in EEG recordings manually is difficult and time consuming; one often needs to skim through tens or even hundreds of hours of EEG recordings. Therefore, automatic detection of such an activity is of great importance. Another potential usage of EEG signal analysis is in the prediction of epileptic activities before they occur, as this will enable the patients (and caregivers) to take appropriate precautions. In this paper, we first present an overview of seizure detection and prediction problem and provide insights on the challenges in this area. Second, we cover some of the state-of-the-art seizure detection and prediction algorithms and provide comparison between these algorithms. Finally, we conclude with future research directions and open problems in this topic.
\end{abstract}

\section{Review}

\subsection{Introduction}

Epilepsy, which is classified as a neurological disorder that affects the brain, impacts about $2 \%$ of the world population leading to a reduction in their productivity and imposing restrictions on their daily life [1]. Diagnosis of epilepsy is done by analyzing electroencephalogram (EEG) signals, as well as patient behavior. EEG signals have two types: scalp EEG and intracranial EEG (iEEG). Scalp EEG signals are usually collected with electrodes placed on the scalp using some sort of conductive gel after treating the scalp area with light abrasion in order to decrease the impedance resulting from dead skin cells. Commonly, 19 recording electrodes in addition to a ground and system reference are placed on the scalp area according to specifications by the International 10-20 system. However, fewer electrodes are used when the EEG signals are recorded for neonates [2]. Each of these electrodes collects an EEG signal, which is centrally recorded for post-processing. On the other hand, in iEEG, electrodes are placed directly on

\footnotetext{
* Correspondence: fathi_sayed@yahoo.com

${ }^{5}$ KACST-TIC in Radio Frequency and Photonics for the e-Society (RFTONICS), King Saud University, Riyadh 11362, Saudi Arabia

${ }^{6}$ Faculty of Electronic Engineering, Menoufia University, Menouf, 32952, Egypt Full list of author information is available at the end of the article
}

the exposed surface of the brain during a surgery to record electrical activity from the cerebral cortex. For the seizure detection task in the case of epilepsy patients, it is required to analyze these EEG signals towards a decision of the existence, or absence, of an epileptic seizure. If a seizure exists, further analysis could be made for more understanding of seizure behavior. Most of the research work in the field of seizure detection depends on scalp EEG signals, which are acquired with non-invasive techniques. So, we will adopt the term EEG to refer to scalp EEG throughout the paper.

The seizure detection process can be made on a single- or multi-channel basis [3,4]. Single-channel seizure detection requires selecting the channel containing the strongest EEG signal collected from the closest point to the seizure spot. This selection process depends mainly on activity measures evaluated for the different channels instantaneously such as the local variance. A better treatment to the seizure detection issue depends on incorporating the information from all EEG signals available into the seizure detection process through data fusion, or multi-channel processing techniques [5].

Several studies have been conducted on EEG seizure detection $[3,4]$. To perform seizure detection on timedomain waveforms, there is a need to create models for the EEG signals, from which features are extracted, 
because treating the signal as discrete-time sequence is not a robust approach for extracting characterizing features. Some algorithms create models for normal and abnormal EEG signals of the patients and use these models in the training process. New signals are tested against these models leading to the decision of seizure or non-seizure activities. Most of the time-domain seizure detection algorithms are patient specific; that is, they are applied to the patient for which training data are extracted. This is intuitively meaningful as each patient has a different nature for his/her EEG signals.

Efforts have also been made to develop seizure prediction algorithms [6,7]. Note that with seizure prediction, it is possible to alert ambulatory patients or caregivers before seizure occurs and causes injury. In addition, seizure prediction could initiate time-sensitive clinical procedures necessary for characterizing epileptic syndromes. It could, also, help in initiating delivery of therapy early enough to attenuate seizure attack and reduce its duration or may even terminate it completely. The endeavor towards fast and simple seizure prediction algorithms has led to the development of a variety of algorithms which will be covered in this paper.

Signal processing has shown a breakthrough with the evolution of several discrete transforms and signal decomposition techniques, because all of these transforms and decompositions have their unique nature. For example, the discrete Fourier transform (DFT) and discrete wavelet transform (DWT) have found popularity in seizure detection and prediction applications. Similarly, the singular value decomposition (SVD) and empirical mode decomposition (EMD) have also found a role in these applications. Even the principal component analysis (PCA) and the independent component analysis (ICA) have found a pre-processing noise removal role in these applications.

Several attempts have been presented in the literature to classify EEG seizure detection and prediction methods. For example, classification into linear and non-linear methods considers variance-based, correlation-based, and simple power spectrum-based methods as linear methods and all other methods as non-linear methods [8-10]. We did not adopt this classification as most of the reviewed methods in this paper are non-linear techniques. Another classification for seizure detection and prediction methods depends on the type of EEG signals used: whether scalp EEG or iEEG. We mentioned in this paper the type of database used for testing each method and the type of the signals in the database.

Tzallas et al. presented a classification of EEG seizure detection methods into pattern recognition methods, morphological analysis methods, parametric methods, decomposition methods, clustering methods, and data mining methods [11]. In this paper, we review some of the recently developed seizure detection and prediction algorithms along with a comparison study between them adopting another basis for classification of seizure detection and prediction methods depending on the transform domain of operation. It seems that seizure detection and prediction are two different trends, but we look at them from a signal processing perspective with different transform domains. We investigate in our description and classification in this paper the most important seizure detection and prediction algorithms operating in each transform domain. Figure 1 shows a classification of the seizure detection and prediction methods based on the processing domain.

Therefore, we organize the paper as follows. We first begin by the most common methods for seizure detection and prediction: time-domain methods, which are addressed in Section 1.2. Section 1.3 is devoted to frequency-domain seizure detection methods. Waveletdomain seizure detection and prediction methods are covered in Section 1.4. Sections 1.5 and 1.6 are devoted for seizure detection methods that are based on the EMD and the SVD, respectively. Section 1.7 presents the role of ICA and PCA in seizure detection. A comparison study illustrating seizure detection and prediction methods is given in Section 2.

\subsection{Time-domain methods}

In general, local activities of EEG waveforms vary from patient to patient; therefore, seizure detection and prediction algorithms are preferred to be patient specific.

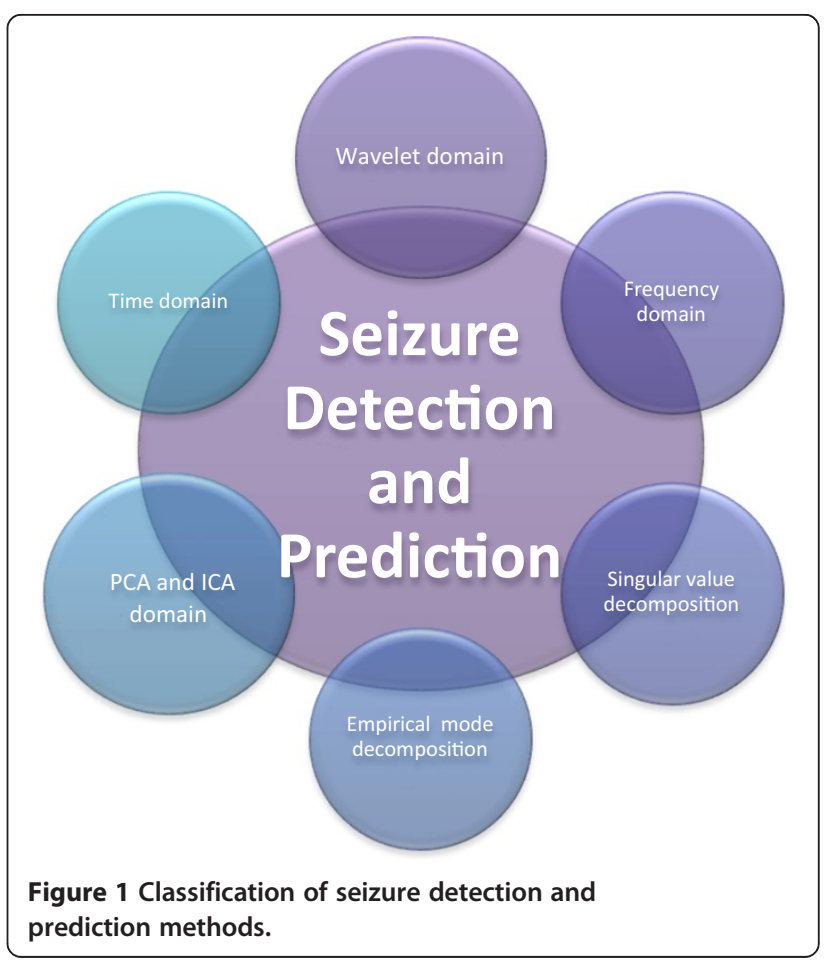


Figure 2 shows an example of an EEG signal including a seizure period. It is clear that there is a difference between seizure and non-seizure intervals. As we are able to differentiate between these intervals visually, timedomain detection and prediction methods attempt to differentiate between them automatically, and evaluate the performance using different metrics such as the sensitivity, specificity, accuracy, and false-positive value. These metrics are defined as follows [12]:

$$
\begin{aligned}
& \text { Sensitivity }=\frac{\mathrm{TP}}{\mathrm{TP}+\mathrm{FN}} \times 100 \\
& \text { Specificity }=\frac{\mathrm{TN}}{\mathrm{TN}+\mathrm{FP}} \times 100 \\
& \text { Accuracy }=\frac{\mathrm{TP}+\mathrm{TN}}{\mathrm{TN}+\mathrm{FP}+\mathrm{TP}+\mathrm{FN}} \times 100
\end{aligned}
$$

False positive value $=\frac{\mathrm{TP}}{\mathrm{TP}+\mathrm{FP}} \times 100$

where true positive (TP) is the number of seizure epochs determined by both algorithm and experienced physicians, false positive (FN) is the number of seizure epochs which are missed by the algorithm but have been determined by experienced physicians, true negative (TN) is the number of non-seizure epochs recognized by both algorithm and experienced physicians, and false positive (FP) is the number of non-seizure epochs recognized as seizure by the algorithm.

\subsubsection{Seizure detection methods}

To detect EEG seizures in time domain, there is a need to analyze discrete time sequences of EEG epochs. This analysis can be accomplished through histograms of the epochs. Runarsson and Sigurdsson presented a simple time-domain seizure detection method that is based on tracing consecutive peaks and minima in the signal segment at hand and estimating the histograms for two variables: the amplitude difference and time separation between peak values as well as minima [13]. The features used for classification of an epoch as a seizure or non-seizure are the estimated values of the histogram bins. The authors used a support vector machine (SVM) classifier for this task and achieved an average sensitivity of about $90 \%$ on self-recorded data.

Another approach to deal with the EEG seizure detection method in time domain is to compute the signal energy during seizure and non-seizure periods. A better treatment to the energy estimation approach is to estimate the energies of the signal sub-bands not the signal as a whole in order to build a more discriminative feature vector. Yoo et al. adopted this approach and presented an eight-channel EEG acquisition system-on-chip (SoC) that can detect and record patient-specific epileptic seizures [14]. They used a bank of seven band-pass filters covering the frequency range from 2 to $26 \mathrm{~Hz}$ on eight channels of the processed EEG signals. Eight highly dynamic analog channels, classification processor, and a 64-KB SRAM have been integrated in the SoC. With this approach, a long-term seizure monitoring and storage

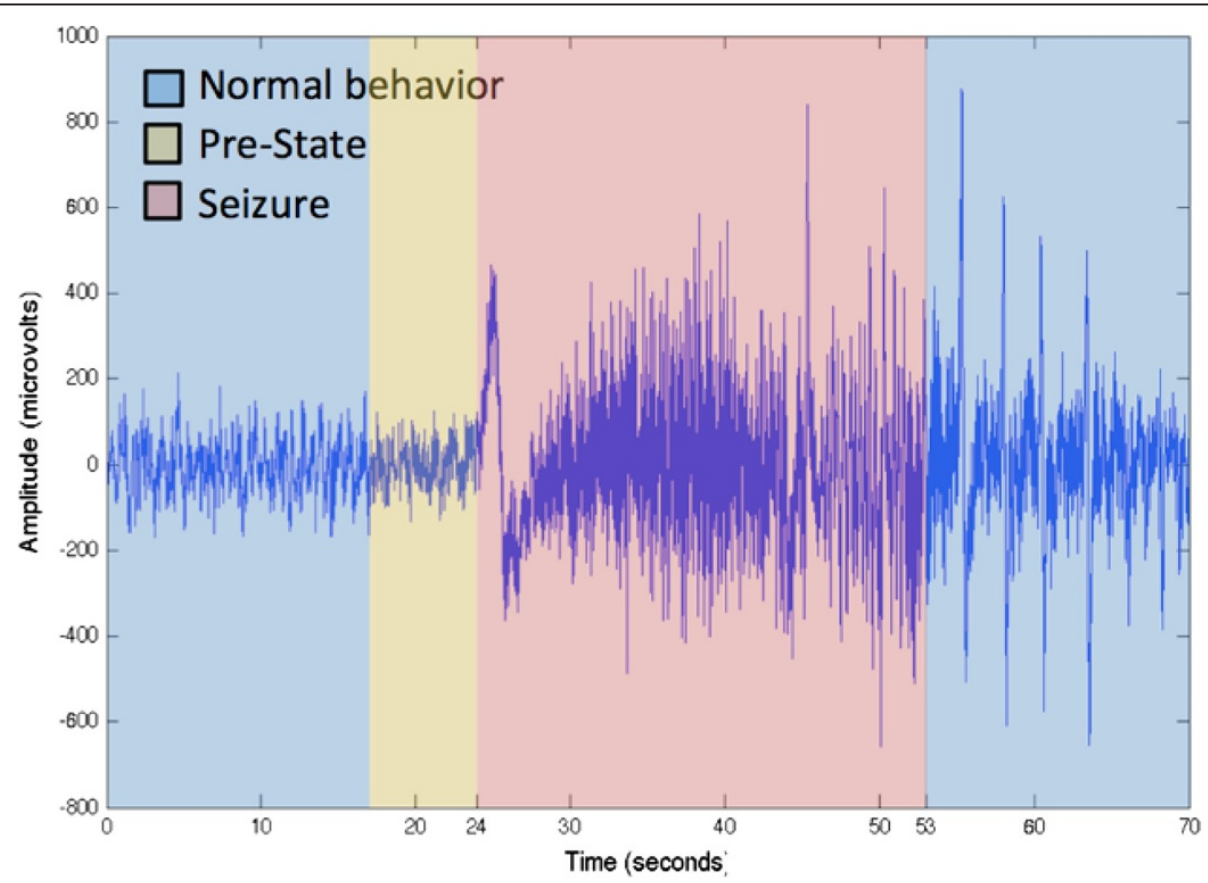

Figure 2 An EEG signal containing a seizure. 
device was built. The authors used an SVM as a classifier with a gain and bandwidth (GBW) controller to perform real-time gain and bandwidth adaptation to analog front end (AFE) in order to keep a high accuracy. This classifier is well suited for the hardware implementation. The SoC was tested on CHB-MIT scalp EEG database [15], and it was verified in the presence of a rapid eye blink giving an accuracy of $84.4 \%$ with $2.03 \mu \mathrm{J} /$ classification energy.

Another approach to deal with time-domain seizure detection is to exploit some discriminating statistics between seizure and non-seizure epochs. Dalton et al. developed a body senor network (BSN) that can monitor and detect epileptic seizures based on statistics extracted from time-domain signals [16]. These statistics include the mean, variance, zero-crossing rate, entropy, and autocorrelation with template signals. For auto-correlation estimation, they adopted a dynamic time warping (DTW) approach for best alignment between the signal segment to be tested and the template signal. The authors presented a network to be embedded in wearable kinematic sensors and an N810 Internet tablet. Data were recorded from the subjects using kinematic sensors such as triaccelerometer, gyroscope, and magnetic sensor for physical activity monitoring. This algorithm was, then, commercially distributed and a BSN was developed on a Mercury platform. The sensitivity of the proposed algorithm for a dataset of 21 seizures was found to be $91 \%$ with a specificity of $84 \%$ and battery lifetime of $10.5 \mathrm{~h}$.

\subsubsection{Seizure prediction methods}

The research work on the issue of time-domain seizure prediction is richer than time-domain seizure detection due to the importance of the seizure prediction problem. We can think of the seizure prediction problem as a detection problem of the pre-ictal state on seizure records. This requires a considerable long inter-ictal state for good prediction results. Similar statistics to those used in seizure detection like the zero-crossing rate can be used for seizure prediction. Zandi et al. used the zerocrossing rate of EEG signal segments to develop a patient-specific seizure prediction method [17,18]. A moving window analysis is used in this method. The histograms of the different window intervals are estimated, and selected histogram bins are used for classification into pre-ictal and inter-ictal states based on comparison with reference histograms. A variational Bayesian Gaussian mixture model has been used for classification. In this method, a combined index for the decisions taken on selected bins is computed and compared with a pre-defined patient-specific threshold to raise an alarm for coming seizures as shown in Figure 3. This method has been tested on $561 \mathrm{~h}$ of scalp EEG containing 86 seizures for 20 patients. It achieved a sensitivity of $88.34 \%$, a false prediction rate of $0.155 \mathrm{~h}^{-1}$, and an average prediction time of $22.5 \mathrm{~min}$.

Aarabi and He [19] developed a time-domain rulebased patient-specific seizure prediction method which consists of three stages: pre-processing, feature extraction, and rule-based decision making. In the pre-processing stage, the iEEG data is filtered using a $0.5-$ to $100-\mathrm{Hz}$ band-pass filter in addition to a $50-\mathrm{Hz}$ notch filter. Then, the filtered signal is segmented into non-overlapping 10-s segments. Five univariate features (correlation entropy, correlation dimension, Lempel-Ziv complexity, noise level, and largest Lyapunov exponent) and one bivariate feature (non-linear independence) were extracted from each segment in the second stage.

Based on the theory of chaos, the correlation dimension (denoted by $v$ ) represents a dimensionality measure of the space having a set of random points; in our case, EEG signals. For an $m$-dimensional space containing a set of $N$ points, we have:

$$
\begin{gathered}
\vec{x}(i)=\left[x_{1}(i), x_{2}(i), \ldots \ldots, x_{m}(i)\right], \\
i=1,2, \ldots ., N
\end{gathered}
$$

The correlation integral $C(\varepsilon)$ can be estimated as [20]:

$$
C(\varepsilon)=\lim _{N \rightarrow \infty} \frac{g}{N^{2}}
$$

where $g$ represents the total number of pairs of signals or points having a distance less than $\varepsilon$. As the number of points increases and tends to infinity and the distance tends to be shorter or close to zero, the correlation integral, in turn, for small values of $\varepsilon$ becomes:

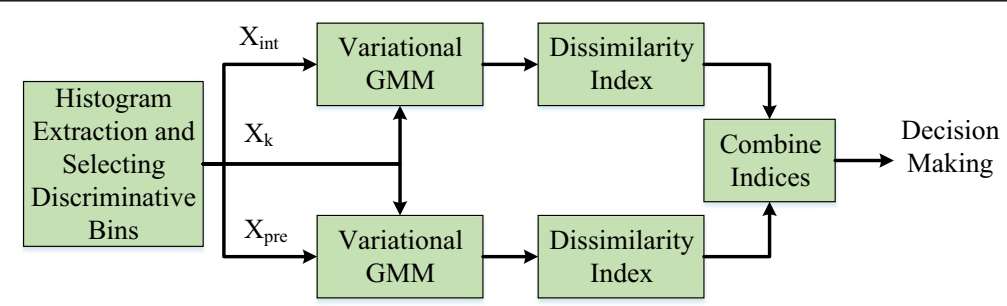

Figure 3 Zandi et al. seizure prediction method. 


$$
C(\varepsilon) \approx \varepsilon^{v}
$$

If a large number of evenly distributed points exists, a $\log -\log$ graph of the correlation integral versus $\varepsilon$ can be used to estimate $v$. For objects with higher dimensions, several ways exist for points to be close to each other, and hence, the number of pairs which are close to each other jumps rapidly for higher dimensions [20].

Correlation entropy is a Kolmogorov entropy variant, which is similar to the mutual information between two sequences of data. Large mutual information between an available data segment and stored segments with specific patterns is an indication that the segment at hand belongs to a dataset with similar characteristics to the stored pattern [21]. The Lempel-Ziv complexity is a measure of randomness of data sequences [22]. It counts the number of data patterns with certain characteristics in data segments. For example, if we find enough short patterns with specific mean, variance, or higher-order statistics in an EEG segment, we can classify this segment as a seizure segment.

The Lyapunov exponent of a dynamical system determines the separation rate of very closely related trajectories. Hence, two signal vectors in the phase space with an initial separation of $\delta \mathbf{Z}_{0}$ will eventually diverge at a rate given by:

$$
|\delta \mathbf{Z}(t)| \approx e^{\lambda t}\left|\delta \mathbf{Z}_{0}\right|
$$

where $\lambda$ is the Lyapunov exponent. This can be achieved if the divergence can be dealt with within the linearized approximation.

The separation rate differs based on the initial separation vector orientation. The maximal Lyapunov exponent can be estimated as [23]:

$$
\lambda=\lim _{t \rightarrow \infty} \lim _{\delta \mathbf{Z}_{0} \rightarrow 0} \frac{1}{t} \ln \frac{|\delta \mathbf{Z}(t)|}{\left|\delta \mathbf{Z}_{0}\right|}
$$

The limit $\delta \mathbf{Z}_{0} \rightarrow 0$ ensures the validity of the linear approximation at any time.

Considering a given segment and feature, if $N$ channels exhibit behaviors like those expected in the feature or segment spatiotemporal profile describing the preictal state of the patient, and if $N$ was greater than a threshold $N_{\text {ch }}$, then this segment is temporarily categorized as a seizure precursor, and hence a flag, labeled as $I$, is raised for the segment as shown in Figure 4. A value is given to this flag by an averaging process for the channels' absolute normalized feature values. The channels selected are those that show similar behavior to the one expected in the spatiotemporal profile of the feature characterizing the pre-ictal state of the patient. The values and location of all flag Is are hence stored and given as input into the feature integrator, which integrates decisions for

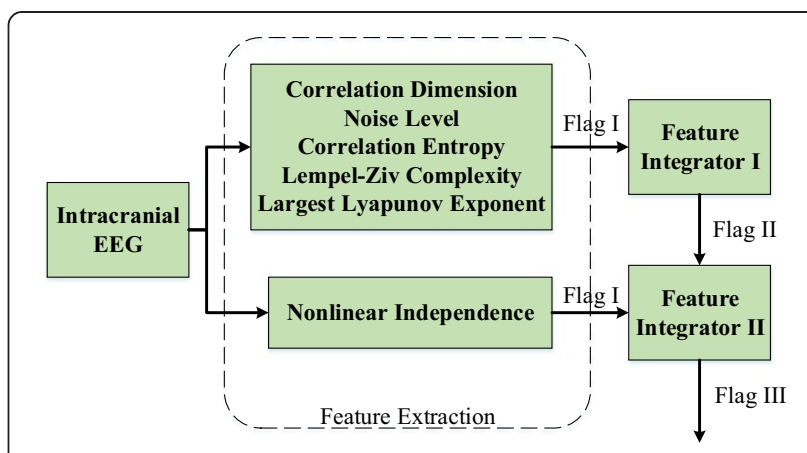

Figure 4 Method of Aarabi and He for seizure prediction.

every segment to accurately locate the seizure precursors. For any signal segment, if $M$ flag $I s$ with values that are higher than a certain significance threshold $T_{\mathrm{c} 1}$ are raised and if $M$ is larger than a certain threshold $N_{F}$, then another flag which is labeled as $I I$ is raised. This flag is determined as the average of the values of the flag Is. Flag IIs ensure a higher likelihood of accurate seizure prediction. Flag IIs are used as input to the feature integrator II to obtain a higher-level decision. This feature integrator integrates flag Is and flag IIs as shown in Figure 4. If both flag $I$ and flag $I I$ are raised, then a flag $I I I$ representing a definitive seizure precursor is also raised.

This method has been evaluated using iEEG data from two patients (frontal, temporal lobe origin) from Freiburg Seizure Prediction EEG (FSPEEG) database with a $256-\mathrm{Hz}$ sampling rate and a total of $58 \mathrm{~h}$, and 10 seizures with 50-min pre-ictal at least [24]. The results demonstrated average sensitivities of $90 \%$ and $96.5 \%$ for patient one and patient two, respectively. The average false prediction rates were $0.06 / \mathrm{h}$ and $0.055 / \mathrm{h}$ for both patients for prediction horizons of 30 and $60 \mathrm{~min}$.

Schelter et al. presented a new method to minimize the false alarms adopting circadian concepts [25]. A circadian rhythm is defined as any biological operation that reveals an endogenous, entrainable oscillation for $24 \mathrm{~h}$. The authors used the output of the mean phase coherent algorithm, which measures the interaction between pairs of EEG signals, as a seizure predictor, which causes an alarm to be raised if it exceeds a certain threshold. It was assumed that the seizures occur while the patient is sleeping. The false alarms display a circadian dependency with most of the seizure prediction algorithms. The seizure predectability is increased during night due to the large number of seizures. Accordingly, threshold adaptation can be used in day and night to enhance predictability. The authors evaluated this method utilizing iEEG data from eight patients and a total of $1400 \mathrm{~h}$, which include 172 seizures and reported a good prediction performance for $40 \%$ of the patients.

Wang et al. proposed an adaptive learning system that interactively learns from the patient and improves its 
seizure predictability over time [26]. It is based on reinforcement learning and online monitoring, in addition to adaptive control theory. In this system, a sliding window size of $10 \mathrm{~min}$ is used to read continuous multichannel EEG data with a $50 \%$ overlap at each move. Then, k-nearest neighbor (KNN) method is adopted for the classification of the windowed epochs to normal or preseizure states based on pre-constructed baselines for both states using pre-specified baseline for normal and preseizure states. Finally, according to the prediction feedbacks, the two baselines are updated. This method was evaluated using iEEG data for five patients having temporal lobe epilepsy. The EEG data consisted of 26 channels with a duration range from 3 to $13 \mathrm{~h}$. This method achieved an accuracy of $70 \%$ compared to $50 \%$ for the Poisson random predictor with a mean interval of $\lambda$ minutes.

Researchers have proved that symptoms like sleep problems or headaches are observable from the analysis of the iEEG. These symptoms can be utilized as a major tool for seizure prediction. Bedeeuzzaman et al. have presented a seizure prediction algorithm with a statistical feature set consisting of mean absolute deviation (MAD) and inter-quartile range (IQR) to predict epileptic seizures [27]. A linear classifier has been used to find the seizure prediction time in pre-ictal iEEGs. A sensitivity of $100 \%$ with zero false-positive rate (FPR) in 12 patients and low values of FPR for the rest were achieved using Freiburg iEEG dataset. Average prediction time varied between 51 and $96 \mathrm{~min}$.

The envelope of the EEG signal can be exploited to distinguish between different activities. Li et al. presented a time-domain method for seizure prediction that is based on spike rate estimation [28]. Morphological operations and averaging filters are applied to transform each signal segment to a train of spikes in a way similar to the process of envelope detection. Based on the spike rate, ictal, inter-ictal, and pre-ictal states can be identified through comparison with a certain threshold. This method was applied on 21 patients from Freiburg database, and it achieved a sensitivity of $75.8 \%$ and an average false-alarm prediction rate of $0.09 / \mathrm{h}$.

Due to the inherent intra- and inter-patient seizure heterogeneities and EEG non-stationarity, it is difficult to measure the modulations of neuronal network interactions using seizure precursors. To solve this problem,
Stamoulis et al. studied the pre-ictal neuro-modulations corresponding to temporal and/or frontal lobe seizures. They used information theoretic features like entropy and mutual information extracted from two frequency ranges (the range that is less than or equal to $100 \mathrm{~Hz}$ and the range that is greater than $100 \mathrm{~Hz}$ ) of awake scalp EEGs [29]. They succeeded to measure pre-ictal modulations at frequencies greater than $100 \mathrm{~Hz}$ with high specificity.

Another approach to process EEG signals in the time domain in order to detect or predict seizure is to create models from the EEG signal segments corresponding to different activities. One of such models is the autoregressive (AR) model, which can be thought of as a data reduction model that transforms the EEG signal segment into few coefficients. Chisci et al. studied the implantation of monitoring and control units on drug-resistant epilepsy patients with AR modeling [30]. They adopted AR modeling with a least-squares parameter estimator for EEG feature extraction in addition to a binary SVM classifier to distinguish between pre-ictal, ictal, and inter-ictal states as shown in Figure 5. This algorithm is computationally simple enabling real-time implementation. Simulation results on the Freiburg database have shown $100 \%$ sensitivity with low false-alarm rate. This is attributed to the regularization strategy of the SVM classifier with Kalman post-processing.

The realization of implantable seizure prediction devices that can be used for alerting the patient and taking an action is a very challenging task. Cellular non-linear networks (CNNs), which represent a paradigm for highspeed computations, can be used for this task. Tetzlaff and Senger presented four different CNN-based approaches for epileptic seizure prediction towards an implantable seizure warning device working on any type of simple time-domain features [31]. This method can be used with any of the abovementioned features. The CNNs have been used in these approaches because they consist of locally coupled dynamical systems that can simulate the non-linear phenomena encountered in physical communication.

\subsection{Frequency-domain methods}

Frequency-domain techniques have been used for EEG seizure detection. Both of the Fourier transform magnitude and phase can be exploited for this purpose. Rana et al. presented a frequency-domain epileptic seizure

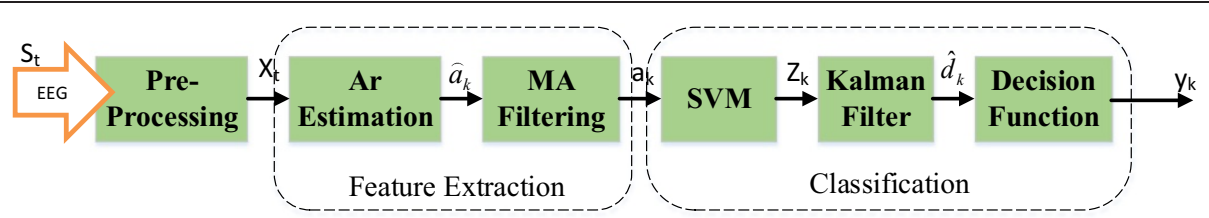

Figure $\mathbf{5}$ Chisci et al. seizure prediction method. 
detection approach depending on the phase-slope index (PSI) of multi-channel EEG signals [32]. If we consider signals $z_{i}[n]$ and $z_{j}[n]$, their cross spectrum is given by:

$$
S_{i j}(f)=E\left[Z_{i}(f) Z_{j}^{*}(f)\right],
$$

where $Z_{i}(f)$ and $Z_{i}(f)$ are the Fourier transforms of $z_{i}$ $[n]$ and $z_{j}[n]$. Hence, the complex coherence is given by:

$$
C_{i j}(f)=\frac{S_{i j}(f)}{\sqrt{S_{i i}(f) S_{j j}(f)}}
$$

An unnormalized PSI metric can be defined using complex coherence as follows:

$$
\tilde{\Psi}_{i j}=\operatorname{Im}\left(\sum_{f \in F} C_{i j}^{*}(f) C_{i j}(f+\delta f)\right),
$$

where $\delta f$ is the frequency resolution and $F$ is the frequency band of interest. We can deduce that $\tilde{\Psi}_{i j}$ measures a weighted sum of the slopes of the phase between $z_{i}$ $[n]$ and $z_{j}[n]$ over the selected band $F[32]$. Normalization with the standard deviation is used to determine whether causal influence from $z_{i}[n]$ to $z_{j}[n]$ is of significant extent or not.

The PSI computes the measure of interaction between two channels. The authors used the PSI metric to distinguish between seizure and normal activities. The detection performance has been evaluated over five patients having different types of epilepsy with 47 seizures in $258 \mathrm{~h}$ of recorded data. The simulation results showed that this algorithm succeeded in the detection of all seizures for four out of five patients, and it achieved a lower false detection rate than two per hour. The results also showed that the channels with strong activity can be determined for each patient.

Khamis et al. used frequency-moment signatures for building a patient-specific seizure detection method [33]. Firstly, experienced electroencephalographs have marked the collected scalp EEG data with seizure events. After that, a filtering process has been performed on the windowed EEG data from electrode differences T6-P4 for the right hemisphere and T5-P3 for the left hemisphere. Power spectral densities of the signals on both hemispheres have been computed and a background removal technique has been used. Moments of these spectra have been used as features for signal classification as seizure or non-seizure. Results reported a sensitivity of $91 \%$ and false-alarm rate of 0.02 false positives per hour.

EEG signals are in general non-linear and non-stationary. So, there is a difficulty to characterize different activities of EEG signals with certain mathematical models. To tackle this problem, Acharya et al. presented a modified method for the detection of normal, pre-ictal, and ictal conditions from recorded EEG signals [34]. This method is based on four entropy features for classification: phase entropy $1\left(S_{1}\right)$, phase entropy $2\left(\mathrm{~S}_{2}\right)$, approximate entropy (ApEn), and sample entropy (SampEn). The phase entropies are estimated from the higher-order spectra of EEG signal epochs as discriminating features for ictal, pre-ictal, and inter-ictal activities. The approximate and sample entropies are logarithmic metrics that determine the closeness and matching between the incoming EEG signal pattern and the recorded templates. These features are extracted from EEG signals and fed to seven different classifiers for comparison: SVM, fuzzy Sugeno classifier (FSC), probabilistic neural network (PNN), KNN, naive Bayes classifier (NBC), decision tree (DT), and Gaussian mixture model (GMM). The obtained results with this method showed the superiority of the Fuzzy classifier, which achieved an accuracy of $98.1 \%$.

\subsection{Wavelet-domain methods}

Wavelets have been widely used in the field of EEG signal analysis, especially for seizure detection and prediction. The wavelet transform in itself can be regarded as some sort of sub-band decomposition, but with downsampling. The wavelet transform can be implemented on analog as well as digital signals. We are more interested in the DWT. The DWT can be implemented with low-pass (LP) and high-pass (HP) filtering in addition to a decimation process, and it must be invertible as shown in Figure 6a [35]. The DWT can be implemented with a single level or multi levels as shown in Figure 6b, c. For the multi-level wavelet decomposition, further decompositions up to the required level are performed on the low-pass branch only. Another implementation of wavelet analysis is the wavelet packet transform, which performs further decomposition on the low-pass and high-pass branches.

The wavelet decomposition and reconstruction filters $H_{0}, H_{1}, G_{0}$, and $G_{1}$ must satisfy the perfect reconstruction (PR) condition. These filters can be obtained by solving the following equation [35]:

$$
\begin{aligned}
Y(z)= & \frac{1}{2}\left\{X_{0}(z)+X_{0}(-z)\right\} G_{0}(z)+\frac{1}{2}\left\{X_{1}(z)+X_{1}(-z)\right\} G_{1}(z) \\
= & \frac{1}{2} X(z)\left\{H_{0}(z) G_{0}(z)+H_{1}(z) G_{1}(z)\right\} \\
& +\frac{1}{2} X(-z)\left\{H_{0}(-z) G_{0}(z)+H_{1}(-z) G_{1}(z)\right\}
\end{aligned}
$$

The main idea of using the wavelet analysis for EEG seizure detection and prediction is extracting discriminating features from appropriate sub-bands to be used for further classification. 


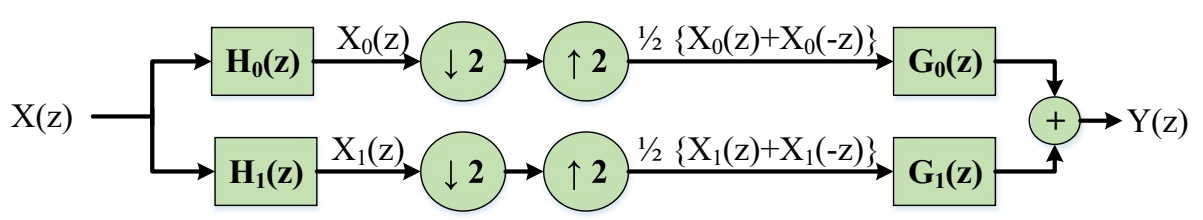

(a)

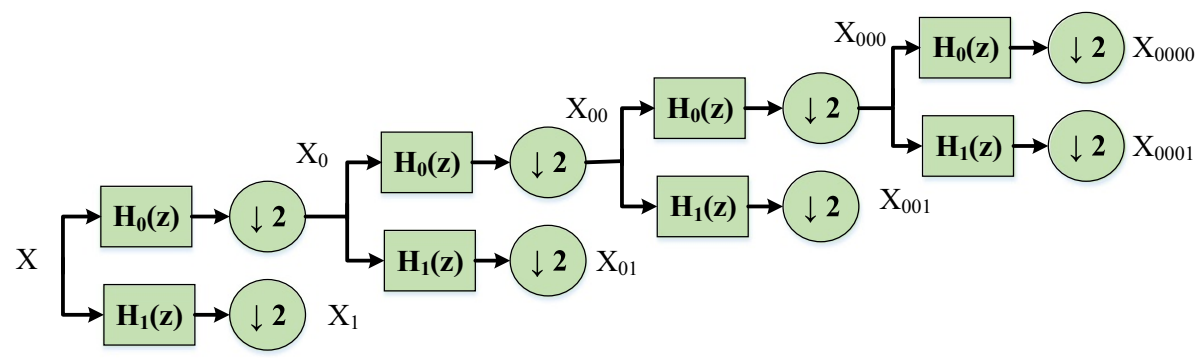

(b)

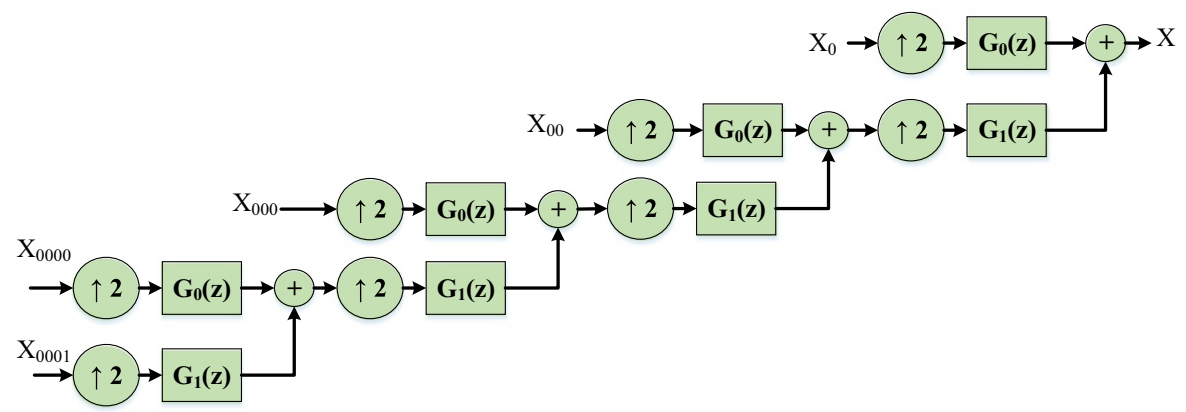

(c)

Figure 6 Wavelet decomposition and reconstruction. The down arrow is down-sampling by 2, and the up arrow is up-sampling by 2. (a) The two-band decomposition-reconstruction filter bank. (b) Multi-level wavelet decomposition tree. (c) Multi-level wavelet reconstruction tree.

\subsubsection{Wavelet-domain seizure detection}

The main challenge in wavelet-based EEG seizure detection is the determination of the appropriate wavelet decomposition level and the selection of the features from certain sub-bands for discrimination between seizure and non-seizure periods. Zhou et al. presented a waveletbased seizure detection method that depends on lacunarity and fluctuation index as features with Bayesian linear discriminant analysis (BLDA) [12]. The lacunarity is a measure of heterogeneity used in fractal analysis. In this method, the authors first decompose the EEG epochs into five wavelet sub-bands. Three sub-bands with scales 3, 4, and 5 are chosen for further processing. For feature extraction, lacunarity and fluctuation index are computed within the frequency bands. If $p(m, l)$ denotes the probability of points, whose amplitude is equal to $m, l$ represents the length of EEG epochs, $A$ is the minimum amplitude value, and $B$ is the maximum amplitude value, then the equation below holds [12]:

$$
\sum_{m=A}^{B} p(m, l)=1
$$

After calculating,

$$
\begin{aligned}
& M_{1}(l)=\sum_{m=A}^{B} m p(m, l) \\
& M_{2}(l)=\sum_{m=A}^{B} m^{2} p(m, l)
\end{aligned}
$$

The lacunarity can be obtained as:

$$
l a(l)=\frac{M_{2}(l)-\left[M_{1}(l)\right]^{2}}{\left[M_{1}(l)\right]^{2}}
$$

After feature extraction, BLDA is used to classify the seizures. The BLDA tries to minimize the risk associated with the classification decision. It can deal with high 
dimensionality and noisy datasets, assuming a linear relation between targets $t$ and feature vectors $\mathbf{x}$ from one side and additive white Gaussian noise $n$ from the other side in Bayesian regression. Post-processing, such as smoothing, and multi-channel decision fusion are applied to enhance the detection accuracy of the BLDA. The performance of this method was investigated on Freiburg EEG database, and it achieved a $96.25 \%$ sensitivity with $0.13 / \mathrm{h}$ false detection rate.

Another five-level wavelet decomposition method for seizure detection was developed by Liu et al. [36]. This method works on multi-channel iEEG signals. Three wavelet sub-bands are selected for further processing. The extracted features from these sub-bands are the relative amplitude, relative energy, coefficient of variation, and fluctuation index from the selected frequency bands. The coefficient of variation is the ratio between the standard deviation of a decomposed sub-band and the square of its mean. The fluctuation index is a measure of the intensity of a decomposed sub-band. An SVM classifier is used in this approach, and some sort of postprocessing is implemented to enhance the detection performance with smoothing, multi-channel decision fusion, and collar processing. The collar processing is technique aiming to maintain the data continuity during processing. The performance of this method has been tested on $509 \mathrm{~h}$ for 21 epilepsy patients. Experimental results have shown that this method achieved a $94.46 \%$ sensitivity, a $95.26 \%$ specificity, and a $0.58 / \mathrm{h}$ false detection rate on long-term iEEG.

The five-level wavelet decomposition was also adopted by Panda et al. with an SVM classifier for seizure detection from background EEGs [37]. This classifier was tested on a healthy subject with open eyes, a healthy subject with closed eyes, and an epilepsy patient. The extracted features for signal classification are energy, standard deviation, and entropy. The simulation results revealed an accuracy of $91.2 \%$ in seizure activity detection. Khan et al. proposed a similar approach for seizure detection, but with relative energy and a normalized coefficient of variation (NCOV) as features [38]. It works on wavelet coefficients acquired in the frequency range of 0 to $32 \mathrm{~Hz}$ as follows:
1) The COV is estimated as:

$$
\mathrm{COV}=\frac{\sigma^{2}}{\mu^{2}}
$$

where $\sigma^{2}$ is the epoch variance and $\mu$ is the epoch mean.

2) The RCOV is estimated as:

$$
\mathrm{RCOV}=\frac{\operatorname{COV}_{e}(n)}{\operatorname{COV}(l)}
$$

where $\mathrm{COV}_{e}(n)$ is the epoch coefficient of variation and $\operatorname{COV}(l)$ is the background coefficient of variation. 3) The NCOV is evaluated as:

$$
\mathrm{NCOV}=\frac{\sigma^{2}}{\mu_{a}},
$$

where $\mu_{a}$ represents the mean of the absolute values of the wavelet coefficients. The obtained results with the NCOV are better than those obtained using the COV only.

Wang et al. used Neyman-Pearson rules and an SVM classifier for seizure detection as shown in Figure 7 [39]. This method depends on the wavelet coefficients in addition to the ApEn in the wavelet domain as extracted features, and the detection is performed using NeymanPearson rules with an SVM. The approximate entropy is an entropy metric that takes into consideration the ordering of the points of the discrete time sequence at hand, and hence, it is a good measure for the regularity of the data sequence. Simulation results have shown a detection accuracy of $98 \%$ and a false detection rate of $6 \%$.

Zainuddin et al. investigated the use of wavelet neural networks (WNNs) based on wavelet basis functions for seizure detection [40]. Firstly, the wavelet transform of EEG signals is estimated, and maximum, minimum, and standard deviation of the absolute values of the wavelet coefficients in each sub-band are extracted as features. These features are then fed to trained WNNs. The Gaussian, Mexican Hat, and Morlet wavelet activation functions have been investigated for classification. A cross-validation approach have been adopted in the

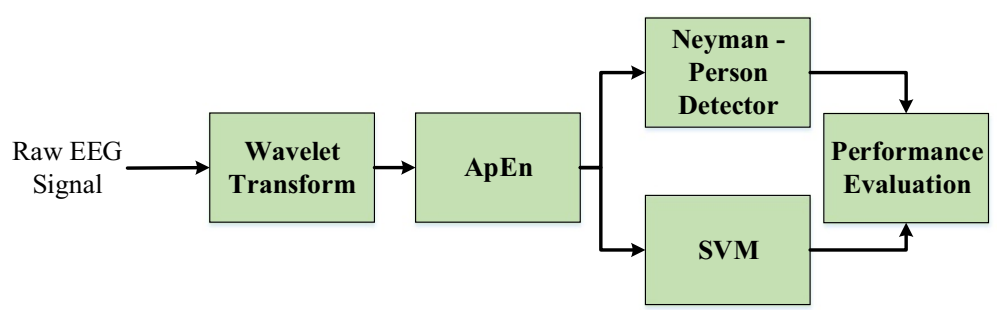

Figure 7 Wang et al. method for seizure detection. 
simulation experiments. Simulation results revealed that the best performance was obtained with WNNs employing a Morlet wavelet activation function with order 4 Daubechies wavelet for feature extraction. The authors have carried simulation experiments on the University of Bonn database for scalp EEG signals [41]. The simulation results reported sensitivity and accuracy up to $98 \%$ with such combination.

Niknazar et al. presented a wavelet-based method for epileptic seizure detection that adopts recurrence quantification analysis (RQA) on EEG recordings and their delta, theta, alpha, beta, and gamma sub-bands extracted through a four-level Daubechies wavelet transform [42]. The RQA is well-suited for non-linear data analysis. It quantifies the number and duration of recurrences of the EEG signals based on phase space trajectories. The phase space is built on estimating a time delay and an embedding dimension, which are the features corresponding to each EEG signal state. The authors adopted an error-correcting output coding (ECOG) classifier for discriminating between three states: healthy, inter-ictal, and ictal. This method achieved an accuracy of $98.67 \%$.

Daou and Labeau presented a wavelet-based approach for EEG signal compression and seizure detection, simultaneously [43]. The compression is performed with the set partitioning in hierarchical trees (SPIHT) algorithm. The SPIHT codes the LP and HP components of the signals with binary sequences. A dictionary is built for these binary sequences of normal and seizure activities. The seizure detection process is performed for each incoming segment by correlation estimation with the binary codes of normal sequences in the dictionary. If low correlation is detected, correlation estimation is performed with binary sequences of the HP components of seizure segments to ensure seizure activity. This method achieved an accuracy of about $90 \%$.

Statistical analysis revealed that the spectra of normal EEG signals follow an inverse attenuation law over the bands of interest, which means that there are self-similar fluctuations in EEG signals over a multiplicity of scales. Based on this idea, Mehta et al. presented a scale-invariant seizure detection method [44]. In this method, the onset is detected, when the scale invariance is lost, which means that the slope of the regression line on a logarithmic plot for the wavelet scales 6 to 2 decreases.

Shoaib et al. presented a low-energy scalable processor for directly analyzing EEG signals acquired through compressive sensing [45]. This work adopts wavelet energy features. It studies the effect of compressive sensing on seizure detection performance showing a reduction of only $4 \%$ in detection sensitivity and an increase of only $0.15 / \mathrm{h}$ in false-alarm rate and $1 \mathrm{~s}$ in latency compared to baseline performance. The main advantage of this method is energy saving archived through compressive sensing.
Zandi et al. presented a wavelet packet real-time seizure detection algorithm working on scalp EEG signals [46]. They developed a patient-specific metric to differentiate between seizure and non-seizure states in the 1to $30-\mathrm{Hz}$ frequency range based on wavelet coefficients of seizure and non-seizure references. A combined seizure index (CSI) is derived from all EEG signal channels depending on the rhythmicity and relative energy of signal epochs for classification as seizure or non-seizure. A majority voting rule is used on the decisions from all channels to generate the CSI and raise an alarm when a seizure is detected. This algorithm was tested on 14 patients having $75.8 \mathrm{~h}$ with 63 seizures, and it achieved a sensitivity of $90.5 \%$, a median detection delay of $7 \mathrm{~s}$, and a false-alarm rate of $0.51 \mathrm{~h}^{-1}$.

\subsubsection{Wavelet-domain seizure prediction}

The same concepts of wavelet signal analysis used for seizure detection can be extended to seizure prediction, but with the target this time as the detection of the preictal state. In general, EEG signals containing seizures are build up of constantly changing bursting levels. This signal nature enables discrimination between different signal activities from wavelet sub-bands. The residual sub-band wavelet entropy (RSWE) can be directly used to estimate the entropy of bursts from the sub-bands as proposed by Paul et al. [47]. The wavelet decomposition equation for an EEG signal using a sliding window of index $m$ is given by:

$$
\begin{aligned}
s(t)= & \sum_{\tau=-\infty}^{\infty} a_{L}^{m}(\tau) \phi\left(2^{-L} t-\tau\right) \\
& +\sum_{l=1}^{L} \sum_{\tau=-\infty}^{\infty} C_{l}^{m}(\tau) \psi\left(2^{-l} t-\tau\right),
\end{aligned}
$$

where $C_{1}^{m}(\tau), C_{2}^{m}(\tau), \ldots, C_{L}^{m}(\tau)$ are the wavelet coefficients. The sequence $\left\{a_{L}^{m}(\tau)\right\}$ is the coarser-resolution signal for a high-level decomposition. The authors experimented lower and higher numbers of levels and found that the five levels are the optimum choice.

The relative wavelet energy (RWE) of the wavelet coefficients is used to derive a sub-band wavelet entropy (SWE) feature. For a sliding window with index $m$, the field potential (FP) is given by [48]:

$$
E_{l}^{m}(\tau)=\left|C_{l}^{m}(\tau)\right|^{2}
$$

The wavelet coefficients total energy is given by:

$$
E_{r m \text { total }}^{m}=\sum_{l} \sum_{\tau} E_{l}^{m}(\tau)
$$

The RWE can be expressed with normalization as: 


$$
p_{l}^{m}(\tau)=\frac{E_{l}^{m}(\tau)}{E_{r m \text { total }}^{m}}
$$

The SWE at scale $l$ can be defined with a probabilistic approach as:

$$
H\left(C_{l}^{m}\right)=\sum_{\tau} p_{l}^{m}(\tau) \log p_{l}^{m}(\tau)
$$

The $l$ th level RSWE of the $n$th frame is given by:

$$
J\left(C_{l}^{m, n}\right)=H\left(C_{l}^{m, n}\right)-\frac{1}{N_{o}} \sum_{n=1}^{N_{o}} H\left(C_{l}^{m, n}\right)
$$

where $C_{l}^{m, n}$ represents the wavelet coefficients of the $n$th frame in the $m$ th window.

For the estimation of the RSWE, a sliding window of 1-min length has been used assuming that the FP is stationary during this window. It was also assumed that the background FP fluctuations stay statistically unchanged for frames of 5-s length during the main sliding window. On the other hand, the FP fluctuations have another component that is statistically variant over the 5-s frames. Hence, an averaging process is performed on the background entropy components estimated in the main window. Consequently, resulting residual entropy corresponds only to the bursting components. A key observation on this approach is that there is an increment in the mean cortical RSWE during the transition from the preictal to inter-ictal period. This increment can be used with an efficient slope change detector and used for early seizure prediction.

Hung et al. developed a very large scale integration (VLSI) setup of wavelet-based seizure prediction algorithm using the correlation dimension $\left(D_{\mathrm{c}}\right)$ and its correlation coefficient [48]. Their system comprises arithmetic functional and control units. The arithmetic functional units are the DWT, correlation dimension, correlation coefficient, and seizure prediction. The DWT of the pre-processed signal is estimated to decompose it into four sub-bands ( 0 to $63 \mathrm{~Hz}, 64$ to $128 \mathrm{~Hz}, 0$ to $1 \mathrm{~Hz}$, and 32 to $64 \mathrm{~Hz}$ ). The higher-frequency sub-bands are then represented in the phase space. The correlation dimension and correlation coefficient are estimated in the phase space as seizure prediction features. The authors evaluated their method utilizing iEEG data from 11 patients of the Freiburg database with $256-\mathrm{Hz}$ sampling rate. Their method achieved an average of $87 \%$ sensitivity, $0.24 / \mathrm{h}$ false prediction rate, and in average a 27-min warning time ahead the ictal.

Chiang et al. developed an online wavelet-domain retraining method to improve the seizure prediction by enlarging the training dataset gradually [49]. Their method is based on the method of Mirowski et al. [50] that uses non-linear interdependence, cross-correlation, difference of Lyapunov exponents, and phase locking. Post-processing is used in this method to reduce the false-alarm rate if two consecutive patterns are classified as pre-ictal. The authors evaluated their method using three datasets: Freiburg database, CHB-MIT database (eight patients), and National Taiwan University Hospital database for scalp EEG (one patient) [51]. This method achieved sensitivities of $74.2 \%$ and $52.2 \%$ on intracranial and scalp databases, respectively. It also achieved an improvement in the sensitivity of off-line training on both databases by $29.0 \%$ and $17.4 \%$, respectively.

Rojas et al. presented a seizure prediction method depending on brain excitability recognized with couplings between low-frequency phases (delta: 0.5 to $33 \mathrm{~Hz}$, theta: 3 to $8 \mathrm{~Hz}$ ) and high-frequency amplitudes (low gamma: 40 to $70 \mathrm{~Hz}$, high gamma 70 to $140 \mathrm{~Hz}$ ) of brain waves [52]. They evaluated this method on 20 patients from EPILEPSIAE scalp EEG database [53]. The EEG data was recorded using either depth or subdural stereotactic electrodes at a sampling rate $1,024 \mathrm{~Hz}$ with a total of 267 seizures and more than $3,400 \mathrm{~h}$ of inter-ictal activities. They found that in $50 \%$ of the cases, their predictor performed better than the random predictor, which is based on Poisson process, with an average sensitivity $98.9 \%$, false rate of $1.84 / \mathrm{h}$, and pre-ictal window length of $10 \mathrm{~min}$.

Gadhoumi et al. presented an iEEG-based seizure prediction method based on measuring the similarity with a reference state as shown in Figure 8 [54]. In the validation of this method, 1,565 h of continuous iEEG data for 17 patients having mesial temporal lobe epilepsy were used. These recordings have 175 seizures. Distance, inclusion, and persistence features are extracted in this method from the continuous wavelet transform of EEG recordings to discriminate between pre-ictal and interictal states. The distance is a Euclidean distance between the center energy and the rest of energy points computed from the wavelet coefficients. Inclusion is the percentage of energy points with entropy confined to a pre-specified distance. Inclusion represents the time interval in which the maximum number of successive 2-s points has energy and entropy profiles confined to the pre-specified distance. An SVM classifier has been used in the signal discrimination and a comparison has been performed with a random statistical predictor. Simulation results have shown a sensitivity of $85 \%$ and a false-alarm rate of $0.35 / \mathrm{h}$. The authors came to a conclusion that state similarity measures can be used for seizure prediction above chance in $41 \%$ of the patients.

Gadhoumi et al. also presented a method to discriminate between pre-ictal and inter-ictal states using features extracted from the high-frequency sub-bands of iEEG signals [55]. The selected features are the wavelet energy and entropy extracted from different signal epochs. The 


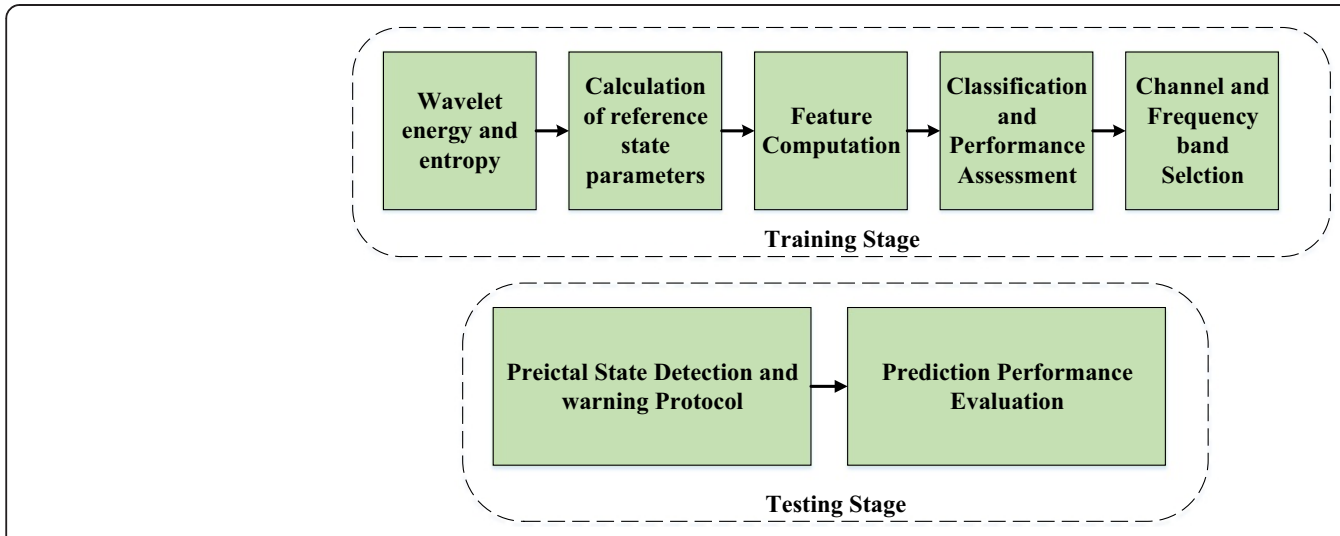

Figure 8 Gadhoumi et al. method showing training and testing stages.

estimated energy and entropy are compared to reference energy and entropy for pre-defined signals with pre-state and seizure activities, and based on distance metrics, the decisions are taken from different channels and fused. Sensitivity and false-alarm rate evaluation revealed a sensitivity of about $80 \%$ and a false positive rate ranging from 0.09 to 0.7 for different patients.

Wang et al. presented a wavelet-based online adaptive seizure prediction system [56]. This system adopts Lyapunov exponent, correlation dimension, Hurst exponent, and entropy features extracted from the wavelet transform of EEG recordings. It adopts also a KNN classifier. The adaptation is performed in this system with a reinforcement learning algorithm. A 150-min prediction horizon used on ten patients and the system achieved the best prediction results of $73 \%$ sensitivity and $67 \%$ specificity.

Soleimani et al. presented a simple and fast adaptive online method for the detection of pre-ictal patterns depending on multiple features as illustrated in Figure 9 [57]. These features are time domain and wavelet domain. The time-domain features are the curve length, the average energy, non-linear energy, six-order power at time $n$, kurtosis, skewness, and variance. The waveletdomain feature include mean of absolute values, average power, standard deviation, absolute mean of sub-bands

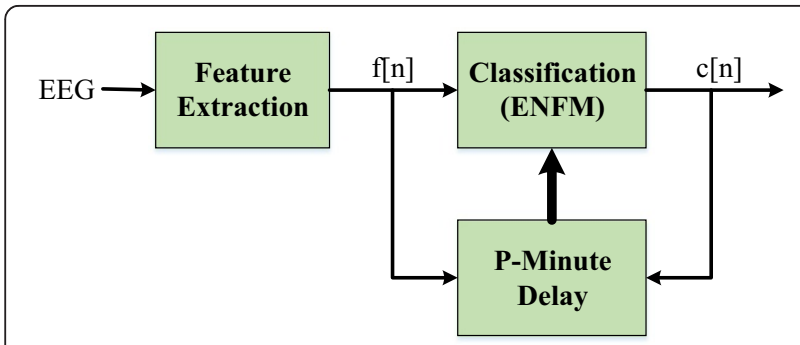

Figure 9 Soleimani et al. seizure prediction method. of a fifth level Daubechies wavelet transform. In this method, a neuro-fuzzy model is used for combined features learning in an adaptive manner. An adaptive tuning process is used in the classifier operation to build a personal seizure predictor. Freiburg database of intracranial recordings for 21 patients has been used with this classifier. Simulation results revealed that on-line adaptive seizure prediction achieves better results than offline non-adaptive seizure prediction. The percentage of prediction was $99.52 \%$, and the FPR was $0.1417 / \mathrm{h}$.

It is possible to use some time-domain features with wavelet-domain features to enhance the detectability of seizures. Costa et al. have selected 14 features for efficient seizure prediction from EEG recordings [58]. These features include energy estimated from time-domain signals, energy variation, energy level, non-linear statistics, and sub-band energies extracted from the wavelet subbands. They used a neural network classifier for signal classification into four states: pre-ictal, ictal, inter-ictal, and post-ictal. They achieved an average specificity of $99 \%$, an average sensitivity of $83 \%$, and an average accuracy of $96 \%$ on patient records from Freiburg database. Moghim and Corne compared the Costa et al. results with multi-class SVM and evolved neural network classifiers [59]. They carried out this comparative study on one patient (patient 2: $135 \mathrm{~min}, 3$ seizures, 30 to $40 \mathrm{~min}$ before and $10 \mathrm{~min}$ after each seizure) from Freiburg database, and they reported that 8- to 10-min detection before the onset can be achieved with reasonable specificity and sensitivity.

\subsection{Empirical mode decomposition}

The EMD is a signal decomposition, which transforms a signal into a group of intrinsic mode functions (IMFs). For EEG seizure detection, these IMFs show different behavior with normal and abnormal activities in the signals. Features can also be extracted from the IMFs and 
tested for seizure detection and prediction. The steps to extract these IMFs from an original signal $x(t)$ are summarized below [60]:

1. Determine the signal envelope by maxima and minima point estimation and interpolate between them.

2. The local mean, $m(t)$ is estimated using the envelope as follows:

$m(t)=\frac{e_{\min }(t)+e_{\max }(t)}{2}$

where $e_{\min }(t)$ and $e_{\max }(t)$ are the envelope minimum and maximum values respectively.

3. The mean is subtracted from the original signal to get $h(t)=x(t)-m(t)$.

4. If $h(t)$ does not satisfy the IMF criteria of a difference of at most 1 between the number of extrema (maxima and minima) and number of zero crossings, we go to step 1 with $h(t)$ as a new input.

5. The obtained $h(t)$ is stored as an IMF in case it satisfies the criteria of an IMF, $c_{i}(t)=h(t)$. Hence, this IMF is removed from the original signal by subtraction, $r(t)=x(t)-c_{i}(t)$, where $i$ is the $i$ th IMF.

6. Similar steps are repeated beginning from step 1 .

The Hilbert transform (HT) is performed on each IMF to get $\tilde{c}_{i}(t)$ provided that $z(t)=c_{i}(t)+j \tilde{c}_{i}(t)$ is analytic. Both amplitude and phase of $z(t)$ are given by:

$$
\begin{aligned}
& A(t)=\sqrt{c_{i}^{2}(t)+\tilde{c}_{i}^{2}(t)} \\
& \phi(t)=\arctan \left[\frac{\tilde{c}_{i}^{2}(t)}{c_{i}^{2}(t)}\right]
\end{aligned}
$$

The instantaneous frequency is estimated as:

$$
\omega=\frac{d \phi(t)}{d t}
$$

\subsubsection{EMD seizure detection}

Eftekhar et al. used the EMD approach for seizure detection [60]. They adopted features such as the frequency rise at the seizure onset with the EMD in a patientspecific manner. Their simulation results have shown that the Hilbert transform can be used to decompose EEG signals into components, from which features can be extracted for seizure onset detection.

Tafreshi et al. evaluated the performance of the EMD in discriminating epileptic seizure data from normal data using means of the absolute of the IMFs as features [61]. They compared this approach for feature extraction with wavelet features using both multi-layer perceptron (MLP) and self-organizing map (SOM) neural networks.
Results have shown that the EMD approach is better in performance than the wavelet approach with success rates up to $95 \%$, and they revealed that four empirical modes are enough to obtain good results. In addition, the results have shown that a window length of 1,500 samples is appropriate to obtain good recognition rates. The MLP networks are superior in performance to the SOM networks.

Orosco et al. presented a seizure detection approach based on the energies of IMFs as discriminating features between seizure and non-seizure activities as shown in Figure 10 [62]. In this approach, the IMF energies are compared with certain thresholds for decision making. It was tested on nine patient records from Freiburg database with invasive nature. The obtained sensitivity and specificity were $56.41 \%$ and $75.86 \%$, respectively, using 39 seizure records.

Guarnizo and Delgado presented a modified EMD approach, in which mutual information is used for feature selection in the EMD domain [63]. These features include the average or instantaneous frequency and amplitude for all EMD components. Higher-order statistics such as the skewness and kurtosis in addition to Shannon's entropy have been selected as features extracted from the energy estimated with the Teager energy operator (TEO) over all EMD components [64-66]. This approach adopts a linear Bayes classifier and it achieved an accuracy of $98 \%$.

Alam and Bhuiyan presented a seizure detection method depending on extracting kurtosis, skewness, largest Lyapunov exponent, variance, approximate entropy, and correlation dimension from the EMD of EEG signals with artificial neural network classifiers $[67,68]$. This method achieved a $100 \%$ sensitivity in seizure detection and has shown a superiority as compared to time-frequency techniques and band-limited techniques in the computational complexity.

Bajaj and Pachori presented an EMD-based seizure detection method to detect focal temporal lobe epilepsy [69]. In this method, they used Hilbert transformation of IMFs which were obtained by an EMD process. Epileptic seizures are then detected based on the instantaneous area estimated from the trace of analytic IMFs of EEG signals. The performance of this epileptic detection method was evaluated on Freiburg database and achieved a sensitivity of $90 \%$, a specificity of $89.31 \%$, and an error detection rate of $24.25 \%$.

The local mean of EMD can be used as a statistical feature for seizure detection. This local mean is obtained by averaging the lower and upper envelopes obtained through interpolation between the local maxima and minima. If these local maxima and minima are not defined as in the case of multivariate signals, another alternative which is the multivariate EMD (MEMD) can be used instead. The MEMD depends on signal projections 


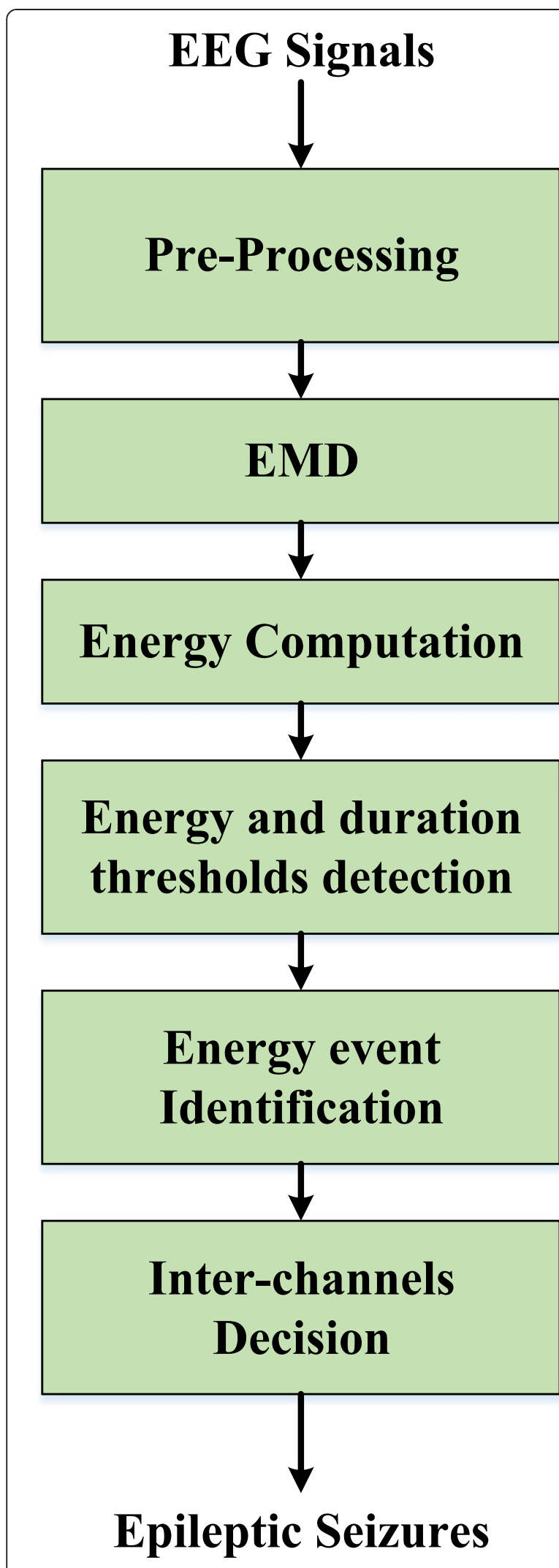

Figure 10 Orosco et al. method for epileptic seizure detection with EMD. along $n$-dimensional spaces to generate $n$-dimensional envelopes, which are then averaged to get the local mean. The projection direction vectors are based on spherical and polar coordinates. To sample the $n$-sphere, quasiMonte Carlo low-discrepancy sequences are exploited for sampling enhancement. This style of sampling is nonuniform but not random. Its objective is to get the most useful samples over a certain space. The other steps of the algorithm to estimate the MEMD are similar to the standard EMD algorithm. The use of the MEMD enables the multi-channel EEG signal decomposition into narrow frequency bands that can be analyzed separately for better detectability of seizures. In addition, the mean frequency of the signal segments can be used as a discriminative feature for seizure detection. This mean frequency can be calculated by applying the Hilbert transform on the resulting IMFs. Rehman et al. investigated the use of the MEMD with the mean frequency as possible features for EEG seizure detection [70], but this proposal needs further studies.

\subsubsection{EMD seizure prediction}

Qi et al. suggested the use of EMD for seizure prediction with a new feature, called IMF-VoE. This feature combines three IMFs obtained from the EMD of an EEG signal in addition to the range variance between upper and lower envelopes (VoE) in the EMD domain [71]. The authors tested this method using $80.4 \mathrm{~h}$ of EEG data containing ten seizures for four patients. This method achieved a sensitivity of $100 \%$ and a false detection rate of $0.16 / \mathrm{h}$. The estimated average time delays were 19.4, 13.2 , and $10.7 \mathrm{~s}$ for false detection rates of $0.16,0.27$, and $0.41 / \mathrm{h}$, respectively, using different thresholds.

\subsection{SVD methods}

The SVD operating on a matrix A decomposes it into matrices $\mathbf{U}, \mathbf{S}$, and $\mathbf{V}[72,73]$ :

$$
\mathbf{A}=\mathbf{U S V}^{\mathrm{T}}
$$

The matrices $\mathbf{U}$ and $\mathbf{V}$ are orthogonal such that $\mathbf{U}^{\mathrm{T}}$ $\mathbf{U}=\mathbf{I}$, and $\mathbf{V}^{\mathrm{T}} \mathbf{V}=\mathbf{I}$. $\mathbf{S}=\operatorname{diag}\left(\sigma_{1}, \ldots, \sigma_{P}\right)$, where $\sigma_{1} \geq \sigma_{2} \geq$ ... $\sigma_{P} \geq 0$ is the matrix of SVs of $\mathbf{A}$. The matrix $\mathbf{U}$ contains the left singular vectors of $\mathbf{A}$, while the matrix $\mathbf{V}$ contains the right singular vectors of $\mathbf{A}$. An important characteristic of the SVD that can be used in EEG seizure detection is that the SVs are not largely affected by little disturbances in the matrix $\mathbf{A}$. This property can be used for the detection of seizure activities taking into consideration the slight variations between seizures $[72,73]$.

Vanrumste et al. developed an SVD-based algorithm for EEG seizure detection [74]. In this algorithm, the EEG is segmented into overlapping epochs, which are formulated as 2-D arrays to allow SVD. Most of the 
signal energy, which differs from seizure to non-seizure activities, is concentrated in the first SVs. After that, an EEG dipole source analysis adopting a single-dipole model is implemented on the EEG segments. This analysis assumes that each spike results from a dipole in the human brain and performs a localization process for the position of this source dipole [75]. This yields dipole parameters in addition to a relative residual energy (RRE). A seizure alarm on an epoch is raised if the SVD indicates a dominant source and the RRE has a low value. The algorithm has been investigated on synthetic EEG signals obtained from two sources, synchronous and asynchronous. In the synchronous case, the main metric is the RRE, and in the asynchronous case, the SVD and RRE are of major concern. The same algorithm has been investigated with real EEG signals having two spikes and an eye-blink artifact. The algorithm has shown that the RRE was always low for all three events.

Shahid et al. presented an algorithm based on SVD for the detection of seizures [76]. In this algorithm, the SVD is applied sequentially on a sliding window of the EEG signal of 1-s width and then $r$ singular values are obtained and used to indicate sudden changes in the signals. EEG recordings of four pediatric patients with 20 seizures have been used to validate this algorithm, and the results indicated the sensitivity of the SVs to the changes in the EEG signals due to epileptic seizure.

\subsection{ICA and PCA methods}

The SVD methods presented in above section adopt a strategy of transforming the 1-D data to a 2-D array, but the PCA and ICA techniques in this section adopt a covariance approach to obtain the PCs. ICA is signal decomposition that transforms a multivariate signal into additive, non-Gaussian, and statistically independent components [77]. The main principle that the ICA depends on is the maximization of the statistical independence between the estimated components. This can be guaranteed through either mutual information minimization or non-Gaussianity maximization. For mutual information minimization, measures like Kullback-Leibler divergence and maximum entropy are exploited. On the other hand, for nonGaussianity maximization, central limit theorem, higherorder statistics such as kurtosis, and negentropy are used [77]. The ICA depends mainly on some pre-processing techniques such as mean removal, whitening, and dimensionality reduction. Both whitening and dimensionality reduction can be achieved with PCA or SVD. Whitening ensures an equal treatment to all dimensions before the algorithm is run.

\subsubsection{Seizure detection}

Both ICA and PCA have been used as signal separation algorithms to enhance the detectability of seizures from
EEG signals by separating artifacts from these signals [78]. Xie and Krishnan proposed a multi-scale PCA method combining both DWT and PCA for de-noising and EEG signal decomposition [79]. They developed an empirical classification (EC) method using spatial and temporal features. This method achieved high classification accuracy.

\subsubsection{Seizure prediction}

Miri and Nasrabadi proposed a seizure prediction technique based on chaos theory and non-linear dynamics to create a return map depending on zero crossings [80]. This method consists of nine steps: raw EEG data recording from six channels, baseline noise reduction through filtering, signal segmentation to non-overlapping 5-min sections, PCA decomposition of the signal, zerocrossing rate estimation for the first PCA component, resampling of the second component based on the estimated zero-crossing rate, creation of a series of samples to construct a Poincare map, feature extraction, and finally estimation of the seizure risk. They evaluated this method with iEEG data from six patients of Freiburg database with $256-\mathrm{Hz}$ sampling rate, 50 -min pre-ictal, and $24 \mathrm{~h}$ of nonseizure data. Their method achieved a $20 \pm 5$-min prediction time, an $86.6 \%$ sensitivity, and a $0.067 / \mathrm{h}$ false-alarm rate.

Williamson et al. presented a seizure prediction method that is based on estimating eigenspectral features from space-delay correlation and covariance matrices of EEG signal blocks with multiple delay scales [81]. An SVM classifier has been used in this method. An averaging process over 15-min windows is used as a post-processing step to get the final prediction score. This method was tested on the recordings of 19 patients from the Freiburg database having at least three seizures. It succeeded in predicting 71 out of 83 seizures. The false prediction rate was $13.8 / \mathrm{h}$

\section{Conclusions}

Table 1 shows a comparison between the different seizure detection and prediction methods. It is clear from this table that most seizure detection and prediction methods adopt time- or wavelet-domain features. For wavelet-domain method, several papers have adopted five-level decomposition for robust feature extraction. Few methods have used hybrid features like time and wavelet-domain features and achieved better performance than using features from one domain only. It is clear also that the EMD is a promising trend for seizure detection and prediction that needs further investigation. Also, we can say that seizure detection and prediction methods based on classifiers are better in performance than methods that do not implement classifiers. Multi- 
Table 1 Summary of seizure detection and prediction methods

\begin{tabular}{|c|c|c|c|c|c|c|c|c|}
\hline Method & $\begin{array}{l}\text { Domain and } \\
\text { algorithm } \\
\text { classification }\end{array}$ & $\begin{array}{c}\text { Single or } \\
\text { multi-channel }\end{array}$ & Database & $\begin{array}{l}\text { Frame } \\
\text { length }\end{array}$ & Features & Classifier & Performance metrics & $\begin{array}{c}\text { Hardware } \\
\text { implementation }\end{array}$ \\
\hline $\begin{array}{l}\text { Runarsson and } \\
\text { Sigurdsson [9] }\end{array}$ & $\begin{array}{c}\text { Time, } \\
\text { detection }\end{array}$ & Single channel & Self-recorded data & $\begin{array}{l}\text { Variable } \\
\text { length } \\
\text { frames }\end{array}$ & $\begin{array}{l}\text { Histogram bin amplitudes for } \\
\text { amplitude difference and } \\
\text { separation time between peaks } \\
\text { and minima }\end{array}$ & SVM & Sensitivity: $90 \%$ & No \\
\hline Yoo et al. [14] & $\begin{array}{l}\text { Time, } \\
\text { detection }\end{array}$ & Multi-channel & MIT database (scalp EEG) & $2 \mathrm{~s}$ & Energies of sub-bands & SVM & $\begin{array}{c}\text { Accuracy: } 84.4 \% \text {, classification } \\
\text { energy } 2.03 \mu \mathrm{J}\end{array}$ & Yes \\
\hline $\begin{array}{l}\text { Dalton et al. } \\
\text { [16] }\end{array}$ & $\begin{array}{l}\text { Time, } \\
\text { detection }\end{array}$ & Single channel & Dataset of 21 seizures & 12 to $25 \mathrm{~s}$ & Signature of seizure & $\begin{array}{l}\text { Template } \\
\text { matching }\end{array}$ & $\begin{array}{l}\text { Sensitivity: } 91 \% \text {, specificity: } \\
84 \% \text {, battery lifetime: } 10.5 \text { h }\end{array}$ & Yes \\
\hline $\begin{array}{l}\text { Zandi et al. } \\
{[17,18]}\end{array}$ & $\begin{array}{l}\text { Time, } \\
\text { prediction }\end{array}$ & Multi-channel & $\begin{array}{l}561 \text { h of scalp EEG with } 86 \\
\text { seizures for } 20 \text { patients }\end{array}$ & $15 \mathrm{~s}$ & Combined index & $\begin{array}{l}\text { Non-linear } \\
\text { SVM with } \\
\text { Gaussian } \\
\text { classifier }\end{array}$ & $\begin{array}{l}\text { Sensitivity: } 88.34 \% \text {, false } \\
\text { prediction rate: } 0.155 \mathrm{~h}^{-1}, \\
\text { average prediction time: } \\
22.5 \mathrm{~min}\end{array}$ & No \\
\hline $\begin{array}{l}\text { Aarabi and } \mathrm{He} \\
{[19]}\end{array}$ & $\begin{array}{l}\text { Time, } \\
\text { prediction }\end{array}$ & Single channel & $\begin{array}{l}316 \mathrm{~h} \text { of } \mathrm{iEEG} \text { data containing } \\
49 \text { seizures for } 11 \text { patients } \\
\text { from Freiburg database }\end{array}$ & $10 \mathrm{~s}$ & $\begin{array}{l}\text { Correlation dimension, } \\
\text { correlation entropy, noise level, } \\
\text { Lempel-Ziv complexity, largest } \\
\text { Lyapunov exponent }\end{array}$ & No classifier & $\begin{array}{c}\text { Average sensitivity: } 79.9 \%, \\
90.2 \% \text { with average false } \\
\text { prediction rate: } 0.17 \text { and } \\
0.11 / \mathrm{h}\end{array}$ & No \\
\hline $\begin{array}{l}\text { Schelter et al. } \\
\text { [25] }\end{array}$ & $\begin{array}{l}\text { Time, } \\
\text { prediction }\end{array}$ & Single channel & $\begin{array}{l}\text { Eight patients with focal } \\
\text { epilepsy }\end{array}$ & $30 \mathrm{~s}$ & Probabilistic threshold & No classifier & Sensitivity: $60 \%$ & No \\
\hline Wang et al. [26] & $\begin{array}{l}\text { Time, } \\
\text { prediction }\end{array}$ & Single channel & Data for five patients & $10 \mathrm{~min}$ & Area under the ROC curve & KNN & Average accuracy: 70\% & No \\
\hline $\begin{array}{l}\text { Bedeeuzzaman } \\
\text { et al. [27] }\end{array}$ & $\begin{array}{l}\text { Time, } \\
\text { prediction }\end{array}$ & Single channel & $\begin{array}{l}21 \text { patients from Freiburg } \\
\text { database (scalp and iEEG) }\end{array}$ & $1 \mathrm{~min}$ & $M A D, I Q R$ & $\begin{array}{l}\text { Binary linear } \\
\text { classifier }\end{array}$ & $\begin{array}{c}\text { Sensitivity: 100\%, FPR: } 0 \text { (for } \\
12 \text { patients), average } \\
\text { prediction time: } 51 \text { to } \\
96 \mathrm{~min}\end{array}$ & No \\
\hline Li et al. [28] & $\begin{array}{l}\text { Time, } \\
\text { prediction }\end{array}$ & Multi channel & $\begin{array}{l}21 \text { patients from Freiburg } \\
\text { database (scalp and iEEG) }\end{array}$ & $5 \mathrm{~s}$ & Spike rate & No classifier & $\begin{array}{l}\text { Accuracy: } 75.8 \% \text {, false } \\
\text { prediction rate: } 0.09\end{array}$ & No \\
\hline Chisci et al. [30] & $\begin{array}{l}\text { Time, } \\
\text { prediction }\end{array}$ & Multi channel & $\begin{array}{l}21 \text { patients from Freiburg } \\
\text { database (scalp and iEEG) }\end{array}$ & $2 \mathrm{~s}$ & AR coefficients & SVM & $\begin{array}{l}\text { Sensitivity: 100\%, average } \\
\text { prediction time: } 60 \mathrm{~min}\end{array}$ & No \\
\hline Rana et al. [32] & $\begin{array}{l}\text { Frequency, } \\
\text { detection }\end{array}$ & Multi-channel & $\begin{array}{c}\text { EEG and ECoG time series, BW: } \\
0.5 \text { to } 100 \mathrm{~Hz}\end{array}$ & 10 to $60 \mathrm{~s}$ & Phase slope index & No classifier & Detection accuracy: 100\% & No \\
\hline $\begin{array}{l}\text { Khamis et al. } \\
\text { [33] }\end{array}$ & $\begin{array}{l}\text { Frequency, } \\
\text { detection }\end{array}$ & Single channel & $\begin{array}{l}12 \text { patients with six data records } \\
\text { (R1 to R6) }\end{array}$ & $32 \mathrm{~s}$ & $\begin{array}{c}\text { Range of frequencies, range } \\
\text { of frequencies used for } \\
\text { background subtraction, number of } \\
\text { points, hemisphere parameters, } \\
\text { moment parameters, artifact } \\
\text { removal parameters, } p \text {-norm power, } \\
\text { cutoff point }\end{array}$ & $\begin{array}{l}\text { Powell's } \\
\text { direction set } \\
\text { method }\end{array}$ & $\begin{array}{l}\text { Sensitivity: } 91 \% \text {, false } \\
\text { positives per hour: } 0.02\end{array}$ & No \\
\hline $\begin{array}{l}\text { Acharya et al. } \\
\text { [34] }\end{array}$ & $\begin{array}{l}\text { Frequency, } \\
\text { detection }\end{array}$ & Single channel & Self-recorded data & $23.6 \mathrm{~s}$ & $\begin{array}{c}\text { Phase entropy } 1\left(S_{1}\right)_{1} \\
\text { phase entropy } 2\left(S_{2}\right)_{1} \\
\text { approximate entropy }(A p E n), \\
\text { and sample entropy (SampEn) }\end{array}$ & $\begin{array}{l}\text { SVM, FSC, } \\
\text { PNN, KNN, } \\
\text { NBC, DT, } \\
\text { and GMM }\end{array}$ & Average accuracy: $98.1 \%$ & No \\
\hline
\end{tabular}


Table 1 Summary of seizure detection and prediction methods (Continued)

\begin{tabular}{|c|c|c|c|c|c|c|c|c|}
\hline Zhou et al. [12] & $\begin{array}{l}\text { Wavelet, } \\
\text { detection }\end{array}$ & Multi-channel & $\begin{array}{c}\text { Freiburg database of } 21 \\
\text { patients; six channels: channels } \\
1,2 \text {, and } 3 \text { near the epileptic } \\
\text { focus, and channels } 4,5 \text {, and } 6 \\
\text { in remote locations }\end{array}$ & $\begin{array}{l}4 \mathrm{~s} \text { without } \\
\text { overlapping }\end{array}$ & $\begin{array}{l}\text { Lacunarity and fluctuation } \\
\text { index on wavelet scales }\end{array}$ & $\begin{array}{l}\text { Bayesian } \\
\text { linear } \\
\text { discriminant } \\
\text { analysis } \\
\text { (BLDA) } \\
\text { classifier }\end{array}$ & $\begin{array}{c}\text { Sensitivity: } 96.25 \% \text {, false } \\
\text { detection rate: } 0.13 / \mathrm{h} \text {, mean } \\
\text { delay time: } 13.8 \mathrm{~s}\end{array}$ & No \\
\hline Liu et al. [36] & $\begin{array}{l}\text { Wavelet, } \\
\text { detection }\end{array}$ & Multi-channel & $\begin{array}{l}\text { Dataset of } 509 \mathrm{~h} \text { from } 21 \\
\text { epileptic patients }\end{array}$ & $4 \mathrm{~s}$ & $\begin{array}{l}\text { Relative energy, relative } \\
\text { amplitude, coefficient of } \\
\text { variation, fluctuation index }\end{array}$ & SVM & $\begin{array}{l}\text { Sensitivity: } 94.46 \% \text {, specificity: } \\
95.26 \% \text {, false detection rate: } \\
0.58 / \mathrm{h}\end{array}$ & No \\
\hline $\begin{array}{l}\text { Panda et al. } \\
{[37]}\end{array}$ & $\begin{array}{l}\text { Wavelet, } \\
\text { detection }\end{array}$ & Single channel & $\begin{array}{l}500 \text { epochs of EEG data from } \\
\text { five different brain activities } \\
\text { (100 signals per epoch) }\end{array}$ & $0.5 \mathrm{~s}$ & $\begin{array}{l}\text { Energy, entropy, standard } \\
\text { deviation }\end{array}$ & SVM & Accuracy: $91.2 \%$ & No \\
\hline Khan et al. [38] & $\begin{array}{l}\text { Wavelet, } \\
\text { detection }\end{array}$ & Single channel & $\begin{array}{l}\text { Self-recorded data for five } \\
\text { patients }\end{array}$ & $25 \mathrm{~s}$ & $\begin{array}{l}\text { Energy and normalized } \\
\text { coefficient of variation }\end{array}$ & $\begin{array}{l}\text { Simple LDA } \\
\text { classifier }\end{array}$ & $\begin{array}{l}\text { Sensitivity: } 83 \% \text {, specificity: } \\
\text { 100\%, accuracy: } 92 \% \text {, overall } \\
\text { precision: } 87 \%\end{array}$ & No \\
\hline Wang et al. [56] & $\begin{array}{l}\text { Wavelet, } \\
\text { detection }\end{array}$ & Single channel & $\begin{array}{l}\text { Changhai Hospital database } \\
\text { (scalp EEG) }\end{array}$ & $\begin{array}{l}70 \text { to } \\
200 \mathrm{~ms}\end{array}$ & Approximate entropy & $\begin{array}{l}\text { Neyman- } \\
\text { Pearson } \\
\text { criteria and } \\
\text { SVM }\end{array}$ & $\begin{array}{l}\text { Detection accuracy: } 98 \% \text {, } \\
\text { false detection rate: } 6 \%\end{array}$ & No \\
\hline $\begin{array}{l}\text { Zainuddin et al. } \\
\text { [40] }\end{array}$ & $\begin{array}{l}\text { Wavelet, } \\
\text { detection }\end{array}$ & Single channel & $\begin{array}{l}\text { University of Bonn database } \\
\text { (scalp EEG) }\end{array}$ & $23.6 \mathrm{~s}$ & $\begin{array}{c}\text { Maximum, minimum, } \\
\text { standard deviation of absolute } \\
\text { wavelet coefficients }\end{array}$ & WNN & $\begin{array}{c}\text { Sensitivity: } 98 \% \text {, accuracy: } \\
98 \%\end{array}$ & No \\
\hline $\begin{array}{l}\text { Niknazar et al. } \\
{[42]}\end{array}$ & $\begin{array}{l}\text { Wavelet, } \\
\text { detection }\end{array}$ & Single channel & $\begin{array}{c}100 \text { single-channel segments } \\
\text { from Bonn University Database } \\
\text { (scalp EEG) }\end{array}$ & $23.6 \mathrm{~s}$ & $\begin{array}{l}\text { Time delay, embedding } \\
\text { dimension }\end{array}$ & ECOG & Accuracy: $98.67 \%$ & No \\
\hline $\begin{array}{l}\text { Daou and } \\
\text { Labeau [43] }\end{array}$ & $\begin{array}{l}\text { Wavelet, } \\
\text { detection }\end{array}$ & Multi-channel & $\begin{array}{l}\text { Nine patients from MIT } \\
\text { database, and nine patients } \\
\text { from Montreal Neurological } \\
\text { Institute database (scalp EEG) }\end{array}$ & $1 \mathrm{~s}$ & SPIHT codes & No classifier & Accuracy: $90 \%$ & No \\
\hline $\begin{array}{l}\text { Mehta et al. } \\
{[44]}\end{array}$ & $\begin{array}{l}\text { Wavelet, } \\
\text { detection }\end{array}$ & Single channel & Self-recorded & $10 \mathrm{~s}$ & $\begin{array}{l}\text { Slope of the regression line } \\
\text { on a logarithmic plot for the } \\
\text { wavelet scales } 6 \text { to } 2\end{array}$ & No classifier & Slope decreases with onset & No \\
\hline $\begin{array}{l}\text { Shoaib et al. } \\
\text { [45] }\end{array}$ & $\begin{array}{l}\text { Wavelet, } \\
\text { detection }\end{array}$ & Multi-channel & MIT database (scalp EEG) & $2 \mathrm{~s}$ & Wavelet energy & SVM & $\begin{array}{l}\text { Sensitivity: } 91 \% \text { to } 96 \% \text {, } \\
\text { latency: } 4.7 \text { to } 5.3 \mathrm{~s} \text {, false- } \\
\text { alarm rate: } 0.17 \text { to } 0.3 / \mathrm{h}\end{array}$ & Yes \\
\hline Zandi et al. [46] & $\begin{array}{l}\text { Wavelet, } \\
\text { detection }\end{array}$ & Multi-channel & $\begin{array}{c}\text { EEG recordings from } 14 \\
\text { patients approximately } 75.8 \mathrm{~h} \\
\text { with } 63 \text { seizures }\end{array}$ & 10 to $40 \mathrm{~s}$ & Combined seizure index & No classifier & $\begin{array}{l}\text { Sensitivity: } 90.5 \%, \text { false } \\
\text { detection rate: } 0.51 \mathrm{~h}^{-1}, \\
\text { median detection delay: } 7 \mathrm{~s}\end{array}$ & No \\
\hline Paul et al. [47] & $\begin{array}{l}\text { Wavelet, } \\
\text { prediction }\end{array}$ & Single channel & Data for rats & $5 \mathrm{~s}$ & RSWE & No classifier & Detection: 90\% & No \\
\hline Hung et al. [48] & $\begin{array}{l}\text { Wavelet, } \\
\text { prediction }\end{array}$ & Multi-channel & $\begin{array}{l}11 \text { patients from Freiburg } \\
\text { database (scalp and iEEG) }\end{array}$ & $1 \mathrm{~h}$ & $\begin{array}{l}\text { Chaos features, wavelet features, } \\
\text { correlation dimension }\end{array}$ & No classifier & $\begin{array}{l}\text { Accuracy: } 87 \% \text {, false } \\
\text { prediction rate: } 0.24 / \mathrm{h}, \\
\text { average warning time: } \\
27 \text { min ahead the ictal }\end{array}$ & Yes \\
\hline
\end{tabular}


Table 1 Summary of seizure detection and prediction methods (Continued)

\begin{tabular}{|c|c|c|c|c|c|c|c|c|}
\hline $\begin{array}{l}\text { Chiang et al. } \\
\text { [49] }\end{array}$ & $\begin{array}{l}\text { Wavelet, } \\
\text { prediction }\end{array}$ & Single channel & $\begin{array}{l}\text { Freiburg database, CHB-MIT } \\
\text { database (eight patients), Na- } \\
\text { tional Taiwan University Hos- } \\
\text { pital database (one patient) }\end{array}$ & $60 \mathrm{~s}$ & Wavelet coherence & SVM & $\begin{array}{l}74.2 \% \text { and } 52.2 \% \text { sensitivity } \\
\text { on intracranial and scalp } \\
\text { databases, sensitivity } \\
\text { improvement by } 29.0 \% \text { and } \\
17.4 \% \text { for both databases }\end{array}$ & No \\
\hline Rojas et al. [52] & $\begin{array}{l}\text { Wavelet, } \\
\text { prediction }\end{array}$ & Single channel & $\begin{array}{c}20 \text { patients (267 seizures, } \\
3,400 \mathrm{~h} \text { ) }\end{array}$ & Up to $22 \mathrm{~h}$ & Cross-frequency coupling & No classifier & Sensitivity: $100 \%$ & No \\
\hline $\begin{array}{l}\text { Gadhoumi } \\
\text { et al. [54] }\end{array}$ & $\begin{array}{l}\text { Wavelet, } \\
\text { prediction }\end{array}$ & Multi-channel & $\begin{array}{c}\text { 1,565 } \mathrm{h} \text { iEEG, } 17 \text { patients with } \\
\text { mesial temporal lobe epilepsy } \\
\text { with } 175 \text { seizures }\end{array}$ & $\begin{array}{l}1 \text { min with } \\
75 \% \\
\text { overlap }\end{array}$ & Distance, inclusion, persistence & SVM & $\begin{array}{l}\text { Sensitivity: }>85 \% \text {, warning } \\
\text { rate: }<0.35 / h \text {, proportion of } \\
\text { time under warning: }<30 \%\end{array}$ & No \\
\hline $\begin{array}{l}\text { Gadhoumi } \\
\text { et al. [55] }\end{array}$ & $\begin{array}{l}\text { Wavelet, } \\
\text { prediction }\end{array}$ & Multi-channel & $\begin{array}{l}\text { Self-recorded dataset for six } \\
\text { patients }\end{array}$ & $2 \mathrm{~s}$, disjoint & Wavelet energy and entropy & $\begin{array}{l}\text { Binary } \\
\text { classifier }\end{array}$ & $\begin{array}{l}\text { Average sensitivity: }>80 \%, \mathrm{FP} \\
\text { rate: from } 0.09 \text { to } 0.7 \mathrm{FP} / \mathrm{h}\end{array}$ & No \\
\hline Wang et al. [56] & $\begin{array}{l}\text { Wavelet, } \\
\text { prediction }\end{array}$ & Multi-channel & $\begin{array}{l}\text { Ten patients (self-recorded } \\
\text { data) }\end{array}$ & $\begin{array}{l}10 \min \\
\text { with } 50 \% \\
\text { overlap }\end{array}$ & $\begin{array}{l}\text { Lyapunov exponent, correlation } \\
\text { dimension, Hurst exponent, } \\
\text { entropy }\end{array}$ & KNN & $\begin{array}{c}\text { Sensitivity: } 73 \% \text {, specificity: } \\
67 \%\end{array}$ & No \\
\hline $\begin{array}{l}\text { Soleimani et al. } \\
\text { [57] }\end{array}$ & $\begin{array}{l}\text { Wavelet and } \\
\text { time, } \\
\text { prediction }\end{array}$ & Single channel & $\begin{array}{l}21 \text { patients Freiburg database } \\
\text { (scalp and iEEG) }\end{array}$ & $10 \mathrm{~s}$ & $\begin{array}{l}\text { Time- and wavelet-domain } \\
\text { features }\end{array}$ & Neuro-fuzzy & $\begin{array}{c}\text { Prediction: } 99.52 \% \text {, FPR: } \\
0.1417 / \mathrm{h}\end{array}$ & No \\
\hline $\begin{array}{l}\text { Moghim and } \\
\text { Corne [59] }\end{array}$ & $\begin{array}{l}\text { Wavelet and } \\
\text { time, } \\
\text { prediction }\end{array}$ & Single channel & $\begin{array}{l}\text { One patient from Freiburg } \\
\text { database (135 min, three } \\
\text { seizures, } 10 \text { min following } \\
\text { each seizure) (scalp and iEEG) }\end{array}$ & $\begin{array}{l}\text { Up to } \\
5 \mathrm{~min}\end{array}$ & $\begin{array}{l}\text { Time-domain energies, } \\
\text { wavelet-domain energies, } \\
\text { correlation dimension, max } \\
\text { Lyapunov exponent }\end{array}$ & $\begin{array}{l}\text { MC-SVM and } \\
\text { EANN }\end{array}$ & $\begin{array}{l}\text { Sensitivity and specificity } \\
\text { with different values } \\
\text { according to simulation } \\
\text { assumptions }\end{array}$ & No \\
\hline $\begin{array}{l}\text { Tafreshi et al. } \\
\text { [61] }\end{array}$ & $\begin{array}{c}\text { EMD, } \\
\text { detection }\end{array}$ & Single channel & $\begin{array}{l}\text { Five patients of Freiburg } \\
\text { database (scalp and iEEG) }\end{array}$ & 4 to $6 \mathrm{~s}$ & $\begin{array}{l}\text { Mean of the absolute of each } \\
\text { IMF, wavelet features }\end{array}$ & $\begin{array}{l}\text { Neural } \\
\text { network }\end{array}$ & Accuracy: 95\% & No \\
\hline $\begin{array}{l}\text { Orosco et al. } \\
\text { [62] }\end{array}$ & $\begin{array}{c}\text { EMD, } \\
\text { detection }\end{array}$ & Single channel & $\begin{array}{c}90 \text { EEG segments acquired for } \\
\text { nine patients }\end{array}$ & $1 \mathrm{~h}$ & Energies of the IMFs & No classifier & $\begin{array}{l}\text { Sensitivity: } 56 \% \text {, specificity: } \\
75 \% \text {, positive predictive } \\
\text { value: } 61 \% \text {, negative } \\
\text { predictive value: } 72 \%\end{array}$ & No \\
\hline $\begin{array}{l}\text { Guarnizo and } \\
\text { Delgado [63] }\end{array}$ & $\begin{array}{c}\text { EMD, } \\
\text { detection }\end{array}$ & Single channel & $\begin{array}{l}\text { Five groups with } 100 \text { single- } \\
\text { channel registers sampled at } \\
173.61 \mathrm{~Hz}\end{array}$ & $\begin{array}{l}\text { Whole } \\
\text { signal }\end{array}$ & $\begin{array}{l}\text { Instantaneous frequency, } \\
\text { amplitude of each EMD component, } \\
\text { skewness, kurtosis, Shannon's entropy }\end{array}$ & $\begin{array}{l}\text { Linear Bayes } \\
\text { classifier }\end{array}$ & Accuracy: $98 \%$ & No \\
\hline $\begin{array}{l}\text { Alam and } \\
\text { Bhuiyan }[67,68]\end{array}$ & $\begin{array}{c}\text { EMD, } \\
\text { detection }\end{array}$ & Single channel & $\begin{array}{l}\text { Bonn University database } \\
\text { (scalp EEG) }\end{array}$ & $23.6 \mathrm{~s}$ & $\begin{array}{l}\text { Skewness, kurtosis, variance, } \\
\text { largest Lyapunov exponent, } \\
\text { correlation dimension, approximate } \\
\text { entropy from intrinsic modes }\end{array}$ & ANN & Accuracy: $100 \%$ & No \\
\hline $\begin{array}{l}\text { Bajaj and } \\
\text { Pachori [69] }\end{array}$ & $\begin{array}{c}\text { EMD, } \\
\text { detection }\end{array}$ & Single channel & $\begin{array}{l}\text { Freiburg database for } 21 \\
\text { patients (scalp and iEEG) }\end{array}$ & $15 \mathrm{~s}$ & Modified central tendency measure & No classifier & $\begin{array}{c}\text { Sensitivity: } 90 \% \text {, specificity: } \\
89.31 \% \text {, error detection rate: } \\
24.25 \%\end{array}$ & No \\
\hline Qi et al. [71] & $\begin{array}{l}\mathrm{EMD}, \\
\text { prediction }\end{array}$ & Single channel & $\begin{array}{l}\text { Pre-surgical epilepsy } \\
\text { monitoring database with } \\
80.4 \mathrm{~h} \text { of EEG data with ten } \\
\text { seizures of four patients }\end{array}$ & $\begin{array}{l}\text { Whole } \\
\text { signal }\end{array}$ & IMF-VoE & No classifier & $\begin{array}{l}\text { Sensitivity: } 100 \% \text {, false } \\
\text { detection rate: } 0.16 / \mathrm{h}, \\
\text { average time delay: } 10.7 \text { to } \\
19.4 \mathrm{~s} \text { are } 19.4 \mathrm{~s}\end{array}$ & No \\
\hline $\begin{array}{l}\text { Vanrumste } \\
\text { et al. [74] }\end{array}$ & $\begin{array}{c}\text { SVD, } \\
\text { detection }\end{array}$ & Single channel & Simulated EEG signals & $10 \mathrm{~s}$ & $\begin{array}{l}\text { Dipole parameters, relative } \\
\text { residual energy (RRE) }\end{array}$ & No classifier & $\begin{array}{l}\text { Visual inspection of } \\
\text { parameters }\end{array}$ & No \\
\hline
\end{tabular}


Table 1 Summary of seizure detection and prediction methods (Continued)

\begin{tabular}{|c|c|c|c|c|c|c|c|c|}
\hline $\begin{array}{l}\text { Shahid et al. } \\
{[76]}\end{array}$ & $\begin{array}{c}\text { SVD, } \\
\text { detection }\end{array}$ & Single channel & $\begin{array}{l}\text { Recordings of four pediatric } \\
\text { patients with } 20 \text { seizures }\end{array}$ & $1 \mathrm{~s}$ & $\begin{array}{l}\text { Largest } r \text {-singular values, Euclidean } \\
\text { distance from the } r \text {-singular } \\
\text { values of a baseline window } \\
\text { located } 1 \mathrm{~h} \text { before the seizure }\end{array}$ & No classifier & Visual inspection of results & No \\
\hline $\begin{array}{l}\text { Xie and } \\
\text { Krishnan [79] }\end{array}$ & $\begin{array}{l}\text { PCA in } \\
\text { wavelet } \\
\text { domain, } \\
\text { detection }\end{array}$ & Single channel & Data for eight patients & $4,097 \mathrm{~s}$ & Adjusted random index (ARI) & $\begin{array}{l}\text { Empirical } \\
\text { classifier }\end{array}$ & ARI up to 1 & No \\
\hline $\begin{array}{l}\text { Miri and } \\
\text { Nasrabadi [80] }\end{array}$ & $\begin{array}{l}\text { PCA } \\
\text { prediction }\end{array}$ & Multi-channel & $\begin{array}{l}\text { Six patients of Freiburg } \\
\text { database (scalp and iEEG) }\end{array}$ & $5 \mathrm{~min}$ & Zero-crossing rate, a statistical index & No classifier & $\begin{array}{c}\text { Prediction time: } 20 \pm 5 \mathrm{~min} \text {, } \\
\text { sensitivity } 86.6 \% \text {, false-alarm } \\
\text { rate: } 0.067 \%\end{array}$ & No \\
\hline $\begin{array}{l}\text { Williamson } \\
\text { et al. [81] }\end{array}$ & $\begin{array}{l}\text { PCA, FFT, } \\
\text { prediction }\end{array}$ & Multi-channel & $\begin{array}{c}\text { Freiburg database (scalp and } \\
\text { iEEG) }\end{array}$ & $15 \mathrm{~s}$ & Eigen spectral features & SVM & $\begin{array}{l}\text { Sensitivity: } 85.5 \% \text {, false } \\
\text { prediction rate: } 0.033 / \mathrm{h}\end{array}$ & No \\
\hline
\end{tabular}




\section{channel techniques are also more superior in perform- ance than single-channel techniques.}

\section{Competing interests}

The authors declare that they have no competing interests.

\section{Author details}

${ }^{1}$ KACST, Riyadh 11442, Kingdom of Saudi Arabia. ${ }^{2}$ Electrical Engineering Department, KACST-TIC in Radio Frequency and Photonics for the e-Society (RFTONICS), King Saud University, Riyadh 11362, Saudi Arabia. ${ }^{3}$ Department of Electrical Engineering, King Saud University, Riyadh 11362, Saudi Arabia. ${ }^{4}$ Institute for Telecommunications Research, University of South Australia, South Australia 5095, Australia. ${ }^{5}$ KACST-TIC in Radio Frequency and Photonics for the e-Society (RFTONICS), King Saud University, Riyadh 11362, Saudi Arabia. ${ }^{6}$ Faculty of Electronic Engineering, Menoufia University, Menouf, 32952, Egypt.

\section{Received: 14 September 2014 Accepted: 25 November 2014} Published: 23 December 2014

\section{References}

1. National Institute of Neurological Disorders and Stroke. http://www.ninds. nih.gov/. Accessed 15 Sept 2014

2. Trans Cranial Technologies Ltd, 10/20 System Positioning Manual (Trans Cranial Technologies Ltd, Kowloon, 2012)

3. UR Acharya, SV Sree, G Swapna, RJ Martis, JS Suri, Automated EEG analysis of epilepsy: a review. Knowl.-Based Syst. 45, 147-165 (2013)

4. DK Binder, SR Haut, Toward new paradigms of seizure detection. Epilepsy Behav. 26(3), 247-252 (2013)

5. B Hunyadi, M Signoretto, WV Paesschen, JAK Suykens, SV Huffel, MD Vos, Incorporating structural information from the multichannel EEG improves patient-specific seizure detection. Clin. Neurophysiol. 123, 2352-2361 (2012)

6. J Rasekhi, MRK Mollaei, M Bandarabadi, CA Teixeira, A Dourado, Preprocessing effects of 22 linear univariate features on the performance of seizure prediction methods. J. Neurosci. Methods 217, 9-16 (2013)

7. PR Carney, S Myers, JD Geyer, Seizure prediction: methods. Epilepsy Behav. 22, S94-S101 (2011)

8. PE McSharry, THe, LA Smith, L Tarassenko, Linear and non-linear methods for automatic seizure detection in scalp electro-encephalogram recordings. Med. Biol. Eng. Comput. 40, 447-461 (2002)

9. PE McSharry, LA Smith, L Tarassenko, Comparison of predictability of epileptic seizures by a linear and a nonlinear method. IEEE Trans. Biomed. Eng. 50(5), 628-633 (2003)

10. V Sakkalis, G Giannakakis, C Farmaki, A Mousas, M Pediaditis, P Vorgia, M Tsiknakis, Absence seizure epilepsy detection using linear and nonlinear EEG analysis methods, in Proceedings of the 35th Annual International Conference of the IEEE EMBS (Osaka, Japan), pp. 6333-6336. 3-7 July 2013

11. AT Tzallas, MG Tsipouras, DG Tsalikakis, EC Karvounis, L Astrakas, S Konitsiotis, M Tzaphlidou, Automated epileptic seizure detection methods: a review study, in Epilepsy - Histological, Electroencephalographic and Psychological Aspects, ed. by D Stevanovic (InTech Europe, Rijeka, 2012), p. 276

12. W Zhou, Y Liu, Q Yuan, X Li, Epileptic seizure detection using lacunarity and Bayesian linear discriminant analysis in intracranial EEG. IEEE Trans. Biomed. Eng. 60(12), 3375-3381 (2013)

13. TP Runarsson, $\mathbf{S}$ Sigurdsson, On-line detection of patient specific neonatal seizures using support vector machines and half-wave attribute histograms, in The International Conference on Computational Intelligence for Modelling, Control and Automation, and International Conference on Intelligent Agents, Web Technologies and Internet Commerce (CIMCA-IAWTIC) (Vienna), pp. 673-677. 28-30 Nov 2005

14. J Yoo, L Yan, D El-Damak, MA Bin Altaf, AH Shoeb, AP Chandrakasan, An 8 channel scalable EEG acquisition SoC with patient-specific seizure classification and recording processor. IEEE J. Solid State Circuits 48(1), 214-228 (2013)

15. AL Goldberger, LAN Amaral, L Glass, JM Hausdorff, P Ivanov, RG Mark, JE Mietus, GB Moody, C-K Peng, HE HE Stanley, PhysioBank, PhysioToolkit, and PhysioNet: components of a new research resource for complex physiologic signals. Circulation 101(23), e215-e220 (2000). http://physionet.org/pn6/chbmit/

16. A Dalton, S Patel, AR Chowdhury, M Welsh, T Pang, S Schachter, G Olaighin, $P$ Bonato, Development of a body sensor network to detect motor patterns of epileptic seizures. IEEE Trans. Biomed. Eng. 59(11), 3204-3211 (2012)

17. AS Zandi, R Tafreshi, M Javidan, GA Dumont, Predicting temporal lobe epileptic seizures based on zero-crossing interval analysis in scalp EEG, in
Proceedings of the 32nd Annual International Conference of the IEEE EMBS (Buenos Aires), pp. 5537-5540. 31 Aug-4 Sept 2010

18. AS Zandi, R Tafreshi, M Javidan, GA Dumont, Predicting epileptic seizures in scalp EEG based on a variational Bayesian Gaussian mixture model of zero-crossing intervals. IEEE Trans. Biomed. Eng. 60(5), 1401-1413 (2013)

19. A Aarabi, B He, A rule-based seizure prediction method for focal neocortical epilepsy. Clin. Neurophysiol. 123(6), 1111-1122 (2012)

20. JC Sprott, G Rowlands, Improved correlation dimension calculation. Int J. Bifurcation. Chaos. 11(7), 1865-1880 (2001)

21. SJ Gu, CP Sun, HQ Lin, Universal role of correlation entropy in critica phenomena. J. Phys. A Math. Theor. 41(2), 1-10 (2008)

22. A Doganaksoy, F Gologlu, On the Lempel-Ziv complexity of sequences. Lect. Notes Comput. Sci 4086, 180-189 (2006)

23. JB Dingwell, Lyapunov Exponents (Wiley Encyclopedia of Biomedical Engineering, New York, 2006)

24. University of Freidberg, Seizure Prediction Project Freidburg (University of Freiburg, 2014). http://epilepsy.uni-freiburg.de/freiburg-seizure-predictionproject/eeg-database. Accessed 15 Sept 2014

25. B Schelter, HF Drentrup, M Ihle, AS Bonhage, J Timmer, Seizure prediction in epilepsy: from circadian concepts via probabilistic forecasting to statistical evaluation, in Proceedings of the IEEE International Conference of IEEE EMBS (Boston, MA, USA), pp. 1624-1627. 30 Aug 30-3 Sept 2011

26. S Wang, WA Chaovalitwongse, S Wong, A novel reinforcement learning framework for online adaptive seizure prediction, in Proceedings of the IEEE International Conference on Bioinformatics and Biomedicine (Hong Kong), pp. 499-504. 18-21 Dec 2010

27. M Bedeeuzzaman, T Fathima, YU Khan, O Farooq, Seizure prediction using statistical dispersion measures of intracranial EEG. Biomed. Signal. Process. Control. 10, 338-341 (2014)

28. S Li, W Zhou, Q Yuan, Y Liu, Seizure prediction using spike rate of intracranial EEG. IEEE Trans. Neural Syst. Rehabil. Eng. 21(6), 880-886 (2013)

29. C Stamoulis, LJ Gruber, DL Schomer, BS Chang, High-frequency neuronal network modulations encoded in scalp EEG precede the onset of focal seizures. Epilepsy Behav. 23(4), 471-480 (2012)

30. L Chisci, A Mavino, G Perferi, M Sciandrone, C Anile, G Colicchio, F Fuggetta, Real-time epileptic seizure prediction using AR models and support vector machines. IEEE Trans. Biomed. Eng. 57(5), 1124-1132 (2010)

31. R Tetzlaff, $\vee$ Senger, The seizure prediction problem in epilepsy: cellular nonlinear networks. IEEE Circuits Syst. Mag. 12(4), 8-20 (2012)

32. P Rana, J Lipor, H Lee, WV Drongelen, MH Kohrman, BV Veen, Seizure detection using the phase-slope index and multichannel ECoG. IEEE Trans. Biomed. Eng. 59(4), 1125-1134 (2012)

33. H Khamis, A Mohamed, S Simpson, Frequency-moment signatures: a method for automated seizure detection from scalp EEG. Clin. Neurophysiol. 124(12), 2317-2327 (2013)

34. UR Acharya, F Molinari, SV Sree, S Chattopadhyay, KH Ng, JS Suri, Automated diagnosis of epileptic EEG using entropies. Biomed. Signal. Process. Control. 7(4), 401-408 (2012)

35. A Prochazka, J Uhlir, PJW Rayner, NJ Kingsbury, Signal Analysis and Prediction (Birkhauser Inc., Switzerland, 1998)

36. Y Liu, W Zhou, Q Yuan, S Chen, Automatic seizure detection using wavelet transform and SVM in long-term intracranial EEG. IEEE Trans. Neural Syst. Rehabil. Eng. 20(6), 749-755 (2012)

37. R Panda, PS Khobragade, PD Jambhule, SN Jengthe, PR Pal, TK Gandhi, Classification of EEG signal using wavelet transform and support vector machine for epileptic seizure diction, in Proceedings of International Conference on Systems in Medicine and Biology (Kharagpur), pp. 405-408. 16-18 Dec 2010

38. YU Khan, N Rafiuddin, O Farooq, Automated seizure detection in scalp EEG using multiple wavelet scales, in Proceedings of the IEEE International Conference on Signal Processing, Computing and Control (ISPCC) (Waknaghat Solan), pp. 1-5. 15-17 March 2012

39. C Wang, C Zhang, J Zou, J Zhang, Performance evaluation for epileptic electroencephalogram (EEG) detection by using Neyman-Pearson criteria and a support vector machine. Physica. A. 391(4), 1602-1609 (2012)

40. Z Zainuddin, LK Huong, O Pauline, On the use of wavelet neural networks in the task of epileptic seizure detection from electroencephalography signals. Proc Comput. Sci. 11(2012), 149-159 (2012)

41. RG Andrzejak, EEG time series download page, in (University of Bonn, 2003). http://epileptologie-bonn.de/cms/upload/workgroup/lehnertz/eegdata.html. Accessed 
42. M Niknazar, SR Mousavi, BV Vahdat, M Sayyah, M Niknazar, SR Mousavi, BV Vahdat, M Sayyah, A new framework based on recurrence quantification analysis for epileptic seizure detection. IEEE J. Biomed Health Inform. 17(3), 572-578 (2013)

43. H Daou, F Labeau, Dynamic dictionary for combined eeg compression and seizure detection. IEEE J. Biomed Health Inform. 18(1), 247-256 (2014)

44. S Mehta, B Onaral, R Koser, Detection of seizure onset using wavelet analysis, in Proceedings of the IEEE Conference of Engineering in Medicine and Biology Society, vol. 2 (Baltimore, MD, USA), pp. 1220-1221. 03-06 Nov 1994

45. M Shoaib, KH Lee, NK Jha, N Verma, A 0.6-107 $\mu \mathrm{W}$ energy-scalable processor for directly analyzing compressively-sensed EEG. IEEE Trans. Circuits Syst I. Reg. Papers 61(4), 1105-1118 (2014)

46. AS Zandi, M Javidan, GA Dumont, R Tafreshi, Automated real-time epileptic seizure detection in scalp EEG recordings using an algorithm based on wavelet packet transform. IEEE Trans. Biomed. Eng. 57(7), 1639-1651 (2010)

47. JS Paul, CB Patel, H Al-Nashash, N Zhang, WC Ziai, MA Mirski, DL Sherman, Prediction of PTZ-induced seizures using wavelet-based residual entropy of cortical and subcortical field potentials. IEEE Trans. Biomed. Eng. 50(5), 640-648 (2003)

48. SH Hung, CF Chao, SK Wang, BS Lin, CT Lin, VLSI implementation for Epileptic Seizure Prediction System based on Wavelet and Chaos Theory, in Proceedings of the IEEE TENCON (Fukuoka), pp. 364-368. 21-24 Nov 2010

49. CY Chiang, NF Chang, TC Chen, HH Chen, LG Chen, Seizure prediction based on classification of EEG synchronization patterns with on-line retraining and post-processing scheme, in Proceedings of the International Conference of the IEEE EMBS (Boston, MA, USA), pp. 7564-7569. 30 Aug-3 Sept 2011

50. PW Mirowski, Y LeCun, D Madhavan, R Kuzniecky, Comparing SVM and convolutional networks for epileptic seizure prediction from intracranial EEG, in IEEE Workshop on Machine Learning for Signal Processing (Cancun), pp. 244-249. 16-19 Oct 2008

51. National Taiwan University Medical Library, 2013. http://ntuml.mc.ntu.edu. tw/Index.action. Accessed 15 Sept 2014

52. CA Rojas, M Valderrama, A Witon, V Navarro, MLV Quyen, Probing cortical excitability using cross-frequency coupling in intracranial EEG recordings: a new method for seizure prediction, in Proceedings of the International Conference of the IEEE EMBS (Boston, MA, USA), pp. 1632-1635. 30 Aug-3 Sept 2011

53. Epilepsiae, in, 2014. http://www.epilepsiae.eu/project_outputs/ european_database_on_epilepsy. Accessed 15 Sept 2014

54. K Gadhoumi, JM Lina, J Gotman, Seizure prediction in patients with mesial temporal lobe epilepsy using EEG measures of state similarity. Clin Neurophysiol. 124(9), 1745-1754 (2013)

55. K Gadhoumi, JM Lina, J Gotman, Discriminating preictal and interictal states in patients with temporal lobe epilepsy using wavelet analysis of intracerebral EEG. Clin. Neurophysiol. 123(10), 1906-1916 (2012)

56. S Wang, WA Chaovalitwongse, S Wong, Online seizure prediction using an adaptive learning approach. IEEE Trans. Knowl. Data Eng. 25(12), 2854-2866 (2013)

57. HB Soleimani, C Lucas, BN Araabi, L Schwabe, Adaptive prediction of epileptic seizures from intracranial recordings. Biomed Signal. Process. Control. 7(5), 456-464 (2012)

58. RP Costa, P Oliveira, G Rodriques, B Direito, A Dourado, Epileptic seizure classification using neural networks with 14 features. Lect Notes Comput Sci 5178, 281-288 (2008)

59. N Moghim, D Corne, Evaluating bio-inspired approaches for advance prediction of epileptic seizures, in Proceedings of the Word Congress on Nature and Biologically Inspired Computing (NaBIC) (Salamanca), pp. 540-545. 19-21 Oct 2011

60. A Eftekhar, F Vohra, C Toumazou, EM Drakakis, K Parker, Hilbert-Huang transform: preliminary studies in epilepsy and cardiac arrhythmias, in Proceedings of the IEEE Biomedical Circuits and Systems Conference, BioCAS (Baltimore, MD, USA), pp. 373-376. 20-22 Nov 2008

61. AK Tafreshi, AM Nasrabadi, AH Omidvarnia, Epileptic seizure detection using empirical mode decomposition, in Proceedings of the IEEE International Symposium on Signal Processing and Information Technology, ISSPIT (Sarajevo), pp. 238-242. 16-19 Dec 2008

62. L Orosco, E Laciar, AG Correa, A Torres, JP Graffigna, An epileptic seizures detection algorithm based on the empirical mode decomposition of EEG, in Proceedings of the International Conference of the IEEE EMBS (Minneapolis, MN, USA), pp. 2651-2654. 3-6 Sept 2009
63. C Guarnizo, E Delgado, EEG single-channel seizure recognition using empirical mode decomposition and normalized mutual information, in Proceedings of the IEEE International Conference on Signal Processing (ICSP) (Beijing), pp. 1-4. 24-28 Oct 2010

64. P Maragos, J Kaiser, T Quatieri, On separating amplitude from frequency modulations using energy operators, in Proceedings of IEEE International Conference on Acoustics, Speech, and Signal Processing, ICASSP-92, vol. 2 (San Francisco, CA, USA), pp. 1-4. 23-26 Mar 1992

65. HM Teager, Some observations on oral air flow during phonation. IEEE Trans Acoust. Speech. Signal. Process. 28(5), 599-601 (1980)

66. HM Teager, SM Teager, Evidence for nonlinear sound production mechanisms in the vocal tract, in Speech Production and Speech Modelling, ed. by WJ Hardcastle, A Marchal (Springer, Amsterdam, 1990), pp. 241-261

67. SMS Alam, MIH Bhuiyan, Detection of epileptic seizures using chaotic and statistical features in the EMD domain, in Proceedings of the India Conference (INDICON) (Hyderabad), pp. 1-4. 16-18 Dec 2011

68. SMS Alam, MIH Bhuiyan, Detection of seizure and epilepsy using higher order statistics in the EMD domain. IEEE J. Biomed Health Inform. 17(2), 312-318 (2013)

69. V Bajaj, RB Pachori, Epileptic seizure detection based on the instantaneous area of analytic intrinsic mode functions of EEG signals. Biomed. Eng. Lett. 3(1), 17-21 (2013)

70. NU Rehman, Y Xia, DP Mandic, Application of multivariate empirical mode decomposition for seizure detection in EEG signals, in Proceedings of the International Conference of the IEEE EMBS (Buenos Aires), pp. 1650-1653. 31 Aug-4 Sept 2010

71. Y Qi, Y Wang, X Zheng, J Zhang, J Zhu, J Guo, Efficient epileptic seizure detection by a combined IMF-VOE feature, in Proceedings of the International Conference of the IEEE EMBS (San Diego, CA, USA), pp. 5170-5173. 28 Aug-1 Sept 2012

72. JM Shieh, DC Lou, MC Chang, A semi-blind watermarking scheme based on singular value decomposition. Comp. Stand. Inter. 28(4), 428-440 (2006)

73. FE Abd El-Samie, Information Security for Automatic Speaker Identification 1st edn. (Springer, Berlin, 2011)

74. B Vanrumste, RD Jones, PJ Bones, Detection of focal epileptiform activity in the EEG: an SVD and dipole model approach, in Proceedings of the Second IEEE Joint EMBS/BMES Conference, vol. 3 (Houston, TX, USA), pp. 2031-2032. 23-26 Oct 2002

75. GV Hoey, B Vanrumste, R Van de Walle, P Boon, I Lemahieu, MB D'Hav, K Vonck, Detection and localization of epileptic brain activity using an artificial neural network for dipole source analysis, in Proceedings of EUSIPCO (Tampere, Finland, 2000), pp. 4-8

76. A Shahid, N Kamel, AS Malik, MA Jatoi, Epileptic Seizure Detection using the singular values of EEG signals, in Proceedings of the IEEE Conference on Complex Medical Engineering (Beijing), pp. 652-655. 25-28 May 2013

77. P Comon, Independent component analysis: a new concept. Signal Process. 36(3), 287-314 (1994)

78. YH Shih, TJ Chen, CH Yang, H Chiueh, Hardware-efficient EVD processor architecture in FastlCA for epileptic seizure detection, in Proceedings of the Signal \& Information Processing Association Annual Summit and Conference (APSIPA ASC) (Hollywood, CA, USA), pp. 1-4. 3-6 Dec 2012

79. S Xie, S Krishnan, Signal decomposition by multi-scale PCA and its applications to long-term EEG signal classification, in Proceedings of the IEEE International Conference on Complex Medical Engineering (Harbin Heilongjiang), pp. 532-537. 22-25 May 2011

80. MR Miri, AM Nasrabadi, A new seizure prediction method based on return map, in Proceedings of the Iranian Conference on BioMedical Engineering (Tehran), pp. 244-248. 14-16 Dec 2011

81. JR Williamson, DW Bliss, DW Browne, JT Narayanan, Seizure prediction using EEG spatiotemporal correlation structure. Epilepsy Behav. 25(2), 230-238 (2012)

\section{doi:10.1186/1687-6180-2014-183}

Cite this article as: Alotaiby et al.: EEG seizure detection and prediction algorithms: a survey. EURASIP Journal on Advances in Signal Processing 2014 2014:183. 